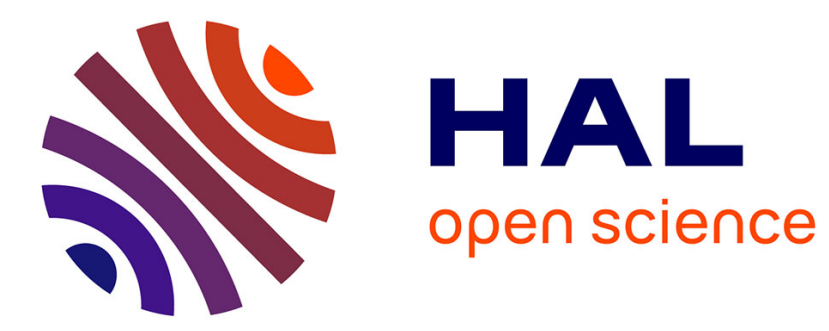

\title{
Estimation of amplitude, phase and unbalance parameters in three phase systems: analytical solutions, efficient implementation and performance analysis
}

Vincent V. Choqueuse, Belouchrani Adel, El Houssin El Bouchikhi, Mohamed Benbouzid

\section{- To cite this version:}

Vincent V. Choqueuse, Belouchrani Adel, El Houssin El Bouchikhi, Mohamed Benbouzid. Estimation of amplitude, phase and unbalance parameters in three phase systems: analytical solutions, efficient implementation and performance analysis. IEEE Transactions on Signal Processing, 2014, 62 (16), pp.4064 - 4076. 10.1109/TSP.2014.2333565 . hal-01058109

\section{HAL Id: hal-01058109 \\ https://hal.science/hal-01058109}

Submitted on 26 Aug 2014

HAL is a multi-disciplinary open access archive for the deposit and dissemination of scientific research documents, whether they are published or not. The documents may come from teaching and research institutions in France or abroad, or from public or private research centers.
L'archive ouverte pluridisciplinaire HAL, est destinée au dépôt et à la diffusion de documents scientifiques de niveau recherche, publiés ou non, émanant des établissements d'enseignement et de recherche français ou étrangers, des laboratoires publics ou privés. 


\title{
Estimation of amplitude, phase and unbal- ance parameters in three-
}

\section{phase systems: analytical solutions, efficient implementation and performance analysis}

V. Choqueuse, Member, IEEE, A. Belouchrani, Member, IEEE, El.H. El Bouchikhi, Student Member, IEEE, M.E.H. Benbouzid, Senior Member, IEEE

\begin{abstract}
This paper focuses on the estimation of the instantaneous amplitude, phase and unbalance parameters in three-phase power systems. Due to the particular structure of three-phase systems, we demonstrate that the Maximum Likelihood Estimates (MLEs) of the unknown parameters have simple closed form expressions and can be easily implemented without matrix algebra libraries. We also derive and analyse the Cramér-Rao Bounds (CRBs) for the considered estimation problem. The performance of the proposed approach is evaluated using synthetic signals compliant with the IEEE Standard C37.118. Simulation results show that the proposed estimators outperform other techniques and reach the CRB under certain conditions.
\end{abstract}

\section{Index Terms}

V. Choqueuse, El.H El Bouchikhi and M.E.H. Benbouzid are with the University of Brest, LBMS (EA 4325), Rue de Kergoat, CS 93837, 29238 Brest Cedex 03, France (e-mail:Vincent.Choqueuse@univ-brest.fr, El-Houssin.Elbouchikhi@univbrest.fr, Mohamed.Benbouzid@univ-brest.fr). A. Belouchrani is with the Ecole Nationale Polytechnique, Algiers 16200, Algeria (e-mail: adel.belouchrani@enp.edu.dz). The authors would like to thank E. Simon for the English language review.

Copyright (c) 2014 IEEE. Personal use of this material is permitted. However, permission to use this material for any other purposes must be obtained from the IEEE by sending a request to pubs-permissions@iee.org. 
Maximum Likelihood Estimation, Cramér-Rao Bounds, Power Systems.

\section{INTRODUCTION}

The analysis of three-phase systems is of major concern in many applications such as power system monitoring, protection and fault detection. In Smart-Grid, voltage and current signals are analysed at substations by Phasor Measurement Units (PMUs). Technically, PMUs estimate the instantaneous amplitude and the instantaneous phase of the voltage or current signal [1]. These measurements are then processed by a Supervisory Control And Data Acquisition (SCADA) system to detect supply-demand mismatches or abnormal events [1]-[3]. On the generation and user sides, current signal analysis (CSA) is also used as a low-cost and non-invasive technique for fault detection in electrical machines [4]. Especially, the instantaneous amplitude and phase are commonly used for the detection of broken rotor bars, eccentricity fault and bearing defects [5]-[8].

The aforementioned applications highlight the importance of Instantaneous Amplitude (IA) and Phase (IP) in modern power systems. In practice, these quantities are unknown and should be estimated from the voltage or current signals. To be compliant with IEEE standards such as the IEEE C37.118 [9], IA and IP estimators must meet certain requirements in terms of accuracy ( $\mathrm{M}$ class) and ability to track fast variations (P class). Within the signal processing community, the most commonly used techniques for the estimation of the IA and IP are based on the analytic signal. The analytic signal is usually computed from the Hilbert Transform or the Fast Fourier Transform [10], [11]. However, these techniques have several limitations as far as power system applications are concerned. Specifically, these techniques require the fulfilment of the Bedrosian condition [12], which can be violated under fast time-varying conditions, and are suboptimal since they do not take into account the particular structure of the electrical signal.

In power systems, the electrical signal is described by three phase components that are phase shifted from each other by $2 \pi / 3$ [9]. From a signal processing perspective, this particular structure provides sufficient information to uniquely identify the IA and IP without the difficulties inherent to monodimensional signals [13]. For instance, under perfectly balanced conditions, it has been proven that the IA and IP can be simply and uniquely identified using a linear transform called the Clarke transform (also called the $\alpha \beta$ transform) [14]-[17]. Nevertheless, despite its low complexity, the Clarke transform does not perform well under voltage or current unbalances. Indeed, it has been demonstrated that the Clarke components are noncircular under unbalanced conditions, which leads to extra oscillations of the IA and IP [17], [18]. Recently, several techniques have been proposed to cope with this difficulty. In [18], [19], the Clarke transform is used jointly with a widely linear adaptive algorithm to estimate 
the instantaneous frequency of the three-phase signal. Although this adaptive algorithm performs well under unbalanced and fast-varying conditions, it requires appropriate tuning and does not provide any estimate of the IA and IP. In [17], an algorithm based on Principal Component Analysis is proposed for IA and IP estimation but its performance highly depends on the accuracy of the covariance matrix estimate. Finally, it should be emphasized that none of the previous techniques is able to quantify the amount of unbalance, which is also of great interest for monitoring purposes [20].

In this paper, we investigate the problem of estimating the IA, IP and unbalance parameters from the voltage (or current) signal under white Gaussian noise assumption. Specifically, the contribution of this paper is threefold.

- We derive closed form solutions for the Maximum Likelihood Estimators (MLEs) of the IA, IP and unbalance parameters.

- We describe a simple low-complexity algorithm for the implementation of these estimators.

- We provide and analyse the Cramér-Rao Bounds of the IA, IP and unbalance parameters.

The remainder of the paper is organized as follows. Section II presents the three-phase signal model and the assumptions. Section III focuses on the MLEs and Section IV deals with the derivation of the Cramér-Rao Bounds. Finally, Section V evaluates the performance of the proposed technique with synthetic signals.

\section{Three-Phase Signal Model}

Hereinafter, bold upper case letters denote matrices, e.g., $\mathbf{X}$; bold lower case letters stand for column vectors, e.g., $\mathbf{y}$, and lower case letters represent scalars. Superscripts $(.)^{T}$ and $(.)^{-1}$ denote the transpose and the inverse of a matrix, respectively. Finally, $\operatorname{Tr}[$.$] is the trace of a matrix, \operatorname{det}($.$) denotes the matrix$ determinant and $\mathbf{I}$ corresponds to the identity matrix.

This study focuses on three-phase systems with amplitude unbalance. Under amplitude unbalance, the three-phase voltages (or currents) are assumed to be perfectly shifted by $2 \pi / 3$ and can be modelled by [9]

$$
y_{k}[n]=d_{k} a[n] \cos (\phi[n]-2 k \pi / 3)+b_{k}[n]
$$

where $y_{k}[n]$ and $b_{k}[n]$ correspond to the electrical signal and noise component of the $k^{\text {th }}$ phase $(k=$ $0,1,2)$, respectively. The time-varying parameters $a[n]>0$ and $\phi[n]$ correspond to the instantaneous amplitude and phase. Several types of profiles for $a[n]$ and $\phi[n]$ are normalized in the IEEE Standard C37.118, such as step change, ramp change, and sine wave modulation [9]. The quantity $d_{k}>0$ corresponds to the unbalance parameter on phase $k$. The ideal case $d_{0}=d_{1}=d_{2}=1$ corresponds 
to a perfectly balanced three-phase system, but is uncommon in practice [21]. For example, voltages in one or two phases can temporary drop for several hundreds of milliseconds due to system faults, heavy load-switching and large motor starting [22]. In the following, we relax the balance assumption by introducing some unbalance between phases as defined in the IEEE standard 1459-2010 [21, 3.2.2]. Without loss of generality, we set $d_{0}=1$ to obtain a well-posed problem since, without any constraint on $d_{k}$, the estimation problem leads to an infinite number of solutions. Using trigonometric identities and matrix notations, (1) can be expressed as

$$
\mathbf{y}[n]=\mathbf{D H x}[n]+\mathbf{b}[n]
$$

where

- $\mathbf{y}[n]$ and $\mathbf{b}[n]$ are $3 \times 1$ column vectors which are defined as

$$
\mathbf{y}[n]=\left[\begin{array}{c}
y_{0}[n] \\
y_{1}[n] \\
y_{2}[n]
\end{array}\right] \quad \mathbf{b}[n]=\left[\begin{array}{c}
b_{0}[n] \\
b_{1}[n] \\
b_{2}[n]
\end{array}\right] .
$$

- D is a $3 \times 3$ diagonal matrix containing the unbalance parameters i.e.

$$
\mathbf{D}=\operatorname{diag}\left(\left[\begin{array}{lll}
1 & d_{1} & d_{2}
\end{array}\right]\right)=\left[\begin{array}{ccc}
1 & 0 & 0 \\
0 & d_{1} & 0 \\
0 & 0 & d_{2}
\end{array}\right]
$$

- $\mathbf{H}$ is a $3 \times 2$ matrix which is defined as

$$
\mathbf{H}=\left[\begin{array}{cc}
1 & 0 \\
-\frac{1}{2} & \frac{\sqrt{3}}{2} \\
-\frac{1}{2} & -\frac{\sqrt{3}}{2}
\end{array}\right]
$$

- $\mathbf{x}[n]$ is a $2 \times 1$ vector containing the direct and quadrature components, i.e.

$$
\mathbf{x}[n]=\mathbf{G}(a[n], \phi[n])=\left[\begin{array}{c}
a[n] \cos (\phi[n]) \\
a[n] \sin (\phi[n])
\end{array}\right]
$$

where $\mathbf{G}(a[n], \phi[n])$ is a multivariate non-linear function.

In order to estimate the unknown parameters, we make use of the following assumptions:

- AS1) The unbalance parameters are assumed to be static over the acquisition time.

- AS2) The additive noise is a zero-mean, white Gaussian noise with covariance matrix. $\sigma^{2} \mathbf{I}$, i.e.

$$
\mathbf{b}[n] \sim \mathcal{N}\left(\mathbf{0}, \sigma^{2} \mathbf{I}\right)
$$


- AS3) The instantaneous amplitude, $a[n]$, phase, $\phi[n]$ and the quadrature components, $\mathbf{x}[n]$, are treated as deterministic unknown parameters.

Although Assumption AS1) may appear somewhat restrictive, it is worth mentioning that the extension to the dynamic case does not present any difficulties ${ }^{1}$. This extension is not discussed here for the sake of brevity. Assumption AS2) is motivated by the Central Limit Theorem and the fact that the Gaussian distribution leads to the largest Cramér-Rao Bound (CRB) [23]. If the Gaussian hypothesis fails to be true, the estimation technique proposed in this paper is still applicable, but it will no longer provide the Maximum Likelihood estimates. Indeed, for non-Gaussian noise, the proposed technique corresponds to the Least Square Estimator (LSE) [24], which is the most natural estimator when no a priori information is available about the noise distribution. Finally, AS3) refers to the Conditional Model and means that the unknown sequence $\mathbf{x}[n]$ is frozen in all the realizations of the data $\mathbf{y}[n]$ [25]. Note that AS3) is less restrictive than the Unconditional Model since it does not require any a priori information about the distribution of $\mathbf{x}[n]$.

Under the above assumptions, the goal of this paper is to estimate $a[n], \phi[n], d_{1}$ and $d_{2}$ from $\mathbf{Y}=$ $[\mathbf{y}[0], \cdots, \mathbf{y}[N-1]]$.

\section{MAXimum LiKelihood Estimation}

In this section, we derive the Maximum Likelihood Estimators (MLEs) of $a[n], \phi[n], d_{1}$ and $d_{2}$. This section is composed of two parts: first, we demonstrate that the MLEs have closed form solutions, then we describe an efficient algorithm for the implementation of these solutions.

\section{A. Closed form estimator}

Let us define

$$
\Omega=\left[a[0], \phi[0], . ., a[N-1], \phi[N-1], d_{1}, d_{2}\right]
$$

the row vector containing the $2 N+2$ unknown parameters. The MLE of $\Omega$ is given by

$$
\widehat{\Omega}=\arg \max _{\Omega} \mathcal{L}(\Omega)
$$

where $\mathcal{L}(\Omega)=\ln (p(\mathbf{Y} ; \Omega))$ is the $\log$-likelihood function of $\mathbf{Y}$ and $p(\mathbf{Y} ; \Omega)$ is the probability density function (pdf) of $\mathbf{Y}$, which is parameterized by $\Omega$. Using AS2) and neglecting the terms that do not depend

\footnotetext{
${ }^{1}$ For dynamic unbalance parameters, the covariance matrix $\widehat{\mathbf{R}}$ in (11) is time-varying and must be replaced by $\widehat{\mathbf{R}}[n]$.
} 
on the unknown parameters, the maximization of $\mathcal{L}(\Omega)$ with respect to $\Omega$ reduces to the minimization of the Least Square criterion [24]

$$
\sum_{n=0}^{N-1}(\mathbf{y}[n]-\mathbf{D H x}[n])^{T}(\mathbf{y}[n]-\mathbf{D H x}[n])
$$

In the following, we decompose the estimation problem into two steps. First, we estimate the unbalance parameters and quadrature components by minimizing (9) with respect to $d_{1}, d_{2}$, and $\mathbf{x}[n]$. Then, we resort to the invariance property of the Maximum Likelihood to estimate $a[n]$ and $\phi[n]$ from the estimate of $\mathbf{x}[n]$.

1) Estimation of $d_{1}$ and $d_{2}$ : Let us denote by $\mathbf{d}=\left[d_{1}, d_{2}\right]^{T}$ the vector containing the unbalance parameters. It can be shown that minimizing (9) with respect to $\mathbf{d}$ is equivalent to the minimization of the following cost function [24]-[26]

$$
\mathcal{J}(\mathbf{d}) \triangleq \operatorname{Tr}[(\mathbf{I}-\mathbf{M}(\mathbf{d})) \widehat{\mathbf{R}}]
$$

where $\widehat{\mathbf{R}}$ is the sample covariance matrix i.e.

$$
\widehat{\mathbf{R}} \triangleq \frac{1}{N} \sum_{n=0}^{N-1} \mathbf{y}[n] \mathbf{y}^{T}[n],
$$

and

$$
\mathbf{M}(\mathbf{d}) \triangleq \mathbf{D H}\left(\mathbf{H}^{T} \mathbf{D}^{2} \mathbf{H}\right)^{-1} \mathbf{H}^{T} \mathbf{D}^{T}
$$

The cost function can be simplified due to the particular structure of $\mathbf{H}$. Indeed, from the definitions of $\mathbf{H}$ and $\mathbf{D}$, we obtain

$$
\mathbf{H}^{T} \mathbf{D}^{2} \mathbf{H}=\frac{1}{4}\left[\begin{array}{cc}
d_{1}^{2}+d_{2}^{2}+4 & -\sqrt{3}\left(d_{1}^{2}-d_{2}^{2}\right) \\
-\sqrt{3}\left(d_{1}^{2}-d_{2}^{2}\right) & 3\left(d_{1}^{2}+d_{2}^{2}\right)
\end{array}\right]
$$

Therefore, the inverse matrix is given by

$$
\begin{aligned}
\left(\mathbf{H}^{T} \mathbf{D}^{2} \mathbf{H}\right)^{-1} & =\frac{1}{3\left(d_{1}^{2}+d_{2}^{2}+d_{1}^{2} d_{2}^{2}\right)} \\
& \times\left[\begin{array}{cc}
3\left(d_{1}^{2}+d_{2}^{2}\right) & \sqrt{3}\left(d_{1}^{2}-d_{2}^{2}\right) \\
\sqrt{3}\left(d_{1}^{2}-d_{2}^{2}\right) & d_{1}^{2}+d_{2}^{2}+4
\end{array}\right]
\end{aligned}
$$

After some computations, it follows that

$$
\mathbf{M}(\mathbf{d})=\frac{1}{d_{1}^{2}+d_{2}^{2}+d_{1}^{2} d_{2}^{2}}\left[\begin{array}{ccc}
d_{1}^{2}+d_{2}^{2} & -d_{1} d_{2}^{2} & -d_{1}^{2} d_{2} \\
-d_{1} d_{2}^{2} & 1-d_{2}^{2} & -d_{1} d_{2} \\
-d_{1}^{2} d_{2} & -d_{1} d_{2} & 1-d_{1}^{2}
\end{array}\right]
$$


From (15), we note that

$$
\mathbf{I}-\mathbf{M}(\mathbf{d})=\frac{1}{d_{1}^{2}+d_{2}^{2}+d_{1}^{2} d_{2}^{2}}\left[\begin{array}{ccc}
d_{1}^{2} d_{2}^{2} & d_{1} d_{2}^{2} & d_{1}^{2} d_{2} \\
d_{1} d_{2}^{2} & d_{2}^{2} & d_{1} d_{2} \\
d_{1}^{2} d_{2} & d_{1} d_{2} & d_{1}^{2}
\end{array}\right] .
$$

Then, it can be checked that

$$
\mathbf{I}-\mathbf{M}(\mathbf{d})=\frac{\mathbf{v}(\mathbf{d}) \mathbf{v}^{T}(\mathbf{d})}{\mathbf{v}^{T}(\mathbf{d}) \mathbf{v}(\mathbf{d})}
$$

where $\mathbf{v}(\mathbf{d})$ is a $3 \times 1$ column vector which is defined as

$$
\mathbf{v}(\mathbf{d}) \triangleq\left[\begin{array}{c}
d_{2} d_{1} \\
d_{2} \\
d_{1}
\end{array}\right]
$$

Using (17) in (10) and the cyclic property of trace, the cost function can be arranged as

$$
\begin{aligned}
\mathcal{J}(\mathbf{d}) & =\frac{\operatorname{Tr}\left[\mathbf{v}(\mathbf{d}) \mathbf{v}^{T}(\mathbf{d}) \widehat{\mathbf{R}}\right]}{\mathbf{v}^{T}(\mathbf{d}) \mathbf{v}(\mathbf{d})} \\
& =\frac{\mathbf{v}^{T}(\mathbf{d}) \widehat{\mathbf{R}} \mathbf{v}(\mathbf{d})}{\mathbf{v}^{T}(\mathbf{d}) \mathbf{v}(\mathbf{d})} .
\end{aligned}
$$

From (20), we observe that the cost function $\mathcal{J}(\mathbf{d})$ reduces to a Rayleigh quotient. A Rayleigh quotient is bounded by [27, Theorem 4.2.2]:

$$
\lambda_{\min } \leq \frac{\mathbf{v}^{T}(\mathbf{d}) \widehat{\mathbf{R}} \mathbf{v}(\mathbf{d})}{\mathbf{v}^{T}(\mathbf{d}) \mathbf{v}(\mathbf{d})} \leq \lambda_{\max }
$$

where $\lambda_{\min }$ and $\lambda_{\max }$ correspond, respectively, to the lowest and highest eigenvalues of $\widehat{\mathbf{R}}$. The lower bound is reached if and only if

$$
\frac{\mathbf{v}(\mathbf{d})}{\|\mathbf{v}(\mathbf{d})\|}=\widehat{\mathbf{u}}
$$

where $\|$.$\| denotes the vector norm and \widehat{\mathbf{u}}$ is the unit-norm eigenvector satisfying $\widehat{\mathbf{R}} \widehat{\mathbf{u}}=\lambda_{\min } \widehat{\mathbf{u}}$. In other words, the entire information about $d_{1}$ and $d_{2}$ is carried by the eigenvector $\widehat{\mathbf{u}}$ associated with the smallest eigenvalue of the sample covariance matrix.

From (22), we can obtain closed form expressions for the MLEs of $d_{1}$ and $d_{2}$. Let us decompose $\widehat{\mathbf{u}}$ as

$$
\widehat{\mathbf{u}} \triangleq\left[\begin{array}{c}
\widehat{u}_{0} \\
\widehat{u}_{1} \\
\widehat{u}_{2}
\end{array}\right]
$$


and let us denote by $\widehat{d}_{1}$ and $\widehat{d_{2}}$, the MLEs of the unbalance parameters. Using (23) and (18) in (22), we obtain the following equations

$$
\begin{aligned}
\widehat{d}_{1} \widehat{d}_{2} & =\widehat{u}_{0}\|\mathbf{v}(\widehat{\mathbf{d}})\| \\
\widehat{d}_{2} & =\widehat{u}_{1}\|\mathbf{v}(\widehat{\mathbf{d}})\| \\
\widehat{d}_{1} & =\widehat{u}_{2}\|\mathbf{v}(\widehat{\mathbf{d}})\| .
\end{aligned}
$$

Therefore, the MLEs of the unbalance parameters are simply given by

$$
\begin{aligned}
& \widehat{d_{1}}=\frac{\widehat{u}_{0}}{\widehat{u}_{1}} \\
& \widehat{d_{2}}=\frac{\widehat{u}_{0}}{\widehat{u}_{2}} .
\end{aligned}
$$

For large $N$, it is shown in Appendix A that $\widehat{d}_{k}(k=1,2)$ is Gaussian distributed. Accordingly, for large $N$, the bias and variance of $\widehat{d}_{k}$ are respectively given by

$$
\begin{aligned}
\operatorname{bias}\left(d_{k}\right) & \approx \frac{\sigma^{2}\|\mathbf{v}(\mathbf{d})\|^{2}}{N} \times \frac{d_{k} \mathbf{C}_{k k}-\mathbf{C}_{0 k}}{w_{k}^{2}} \\
\operatorname{var}\left(\widehat{d}_{k}\right) & \approx \frac{\sigma^{2}\|\mathbf{v}(\mathbf{d})\|^{2}}{N} \times \frac{\mathbf{C}_{00}-2 d_{k} \mathbf{C}_{0 k}+d_{k}^{2} \mathbf{C}_{k k}}{w_{k}^{2}}
\end{aligned}
$$

where $w_{1}=d_{2}, w_{2}=d_{1}, \mathbf{C}_{i j}$ corresponds to the $[i, j]$ element of the $3 \times 3$ matrix

$$
\mathbf{C}=\sum_{k=0}^{1} \frac{\lambda_{k}}{\left(\lambda_{k}-\sigma^{2}\right)^{2}} \mathbf{s}_{k} \mathbf{s}_{k}^{T},
$$

and $\mathbf{s}_{k}(k=0,1)$ are the unit norm eigenvectors associated with the two largest eigenvalues $\lambda_{k}(k=0,1)$ of $\mathbf{R} \triangleq E\left[\mathbf{y}[n] \mathbf{y}^{T}[n]\right]$. From (26), it can be observed that the bias and variance of the estimators go to zero as $\sigma^{2} \rightarrow 0$ or $N \rightarrow \infty$.

2) Estimation of $\boldsymbol{x}[n], a[n]$ and $\phi[n]$ : Let us denote by $\widehat{\mathbf{D}}=\operatorname{diag}\left(\left[\begin{array}{lll}1 & \widehat{d}_{1} & \widehat{d}_{2}\end{array}\right]\right)$ the diagonal matrix containing the estimates of the unbalance parameters. It can be shown that the MLEs of the direct and quadrature components, $\mathbf{x}[n]$, are given by [24]

$$
\widehat{\mathbf{x}}[n]=\left(\mathbf{H}^{T} \widehat{\mathbf{D}}^{2} \mathbf{H}\right)^{-1} \mathbf{H}^{T} \widehat{\mathbf{D}}^{T} \mathbf{y}[n] .
$$

Remark 1: For balanced systems $\left(\widehat{d}_{1}=\widehat{d}_{2}=1\right)$, the MLEs of the direct and quadrature components reduce to

$$
\widehat{\mathbf{x}}[n]=\left[\begin{array}{ccc}
\frac{2}{3} & -\frac{1}{3} & -\frac{1}{3} \\
0 & \frac{\sqrt{3}}{3} & -\frac{\sqrt{3}}{3}
\end{array}\right] \mathbf{y}[n] .
$$

In the power electronics community, this transform is known as the Clarke transform [14]. 
To estimate $a[n]$ and $\phi[n]$ from $\widehat{\mathbf{x}}[n]$, we resort to the invariance property of the MLE [28, Theorem 7.4]. Using this property, the MLEs of the IA and IP are given by

$$
\left[\begin{array}{l}
\widehat{a}[n] \\
\widehat{\phi}[n]
\end{array}\right]=\mathbf{G}^{-1}(\widehat{\mathbf{x}}[n]),
$$

where $\mathbf{G}^{-1}$ (.) corresponds to the inverse of the multivariate non-linear function $\mathbf{G}($.$) in (6). Decomposing$ $\widehat{\mathbf{x}}[n]$ as $\widehat{\mathbf{x}}[n]=\left[\widehat{x}_{\alpha}[n], \widehat{x}_{\beta}[n]\right]^{T}$ and using the definition of $\mathbf{G}($.$) , we obtain$

$$
\begin{aligned}
& \widehat{a}[n]=\sqrt{\widehat{x}_{\alpha}^{2}[n]+\widehat{x}_{\beta}^{2}[n]} \\
& \widehat{\phi}[n]=\arctan \left(\frac{\widehat{x}_{\beta}[n]}{\widehat{x}_{\alpha}[n]}\right) .
\end{aligned}
$$

\section{B. Algorithm Implementation}

The proposed estimation procedure is summarized in Figure 1. From a practical viewpoint, the more difficult task relies on the evaluation of $\widehat{d}_{1}, \widehat{d}_{2}$ and $\widehat{\mathbf{x}}[n]$. Indeed, $\widehat{d}_{1}$ and $\widehat{d}_{2}$ requires the computation of the eigenvector $\widehat{\mathbf{u}}$ and $\widehat{\mathbf{x}}[n]$ requires a matrix inversion. These computations can be performed by specialized mathematical libraries such as LAPACK or the GNU Scientific Library. However, these libraries are designed mainly for large matrices and may produce a lot of computational overhead for small matrices [29]. To reduce computational time and avoid the use of external libraries, we provide in this subsection the analytic expressions of $\widehat{d}_{1}, \widehat{d}_{2}$ and $\widehat{\mathbf{x}}[n]$.

1) Expression of $\widehat{d}_{1}$ and $\widehat{d_{2}}$ : Let us express the $3 \times 3$ symmetric matrix $\widehat{\mathbf{R}}$ as

$$
\widehat{\mathbf{R}}=\left[\begin{array}{lll}
r_{00} & r_{01} & r_{02} \\
r_{01} & r_{11} & r_{12} \\
r_{02} & r_{12} & r_{22}
\end{array}\right]
$$

The eigenvalues of $\widehat{\mathbf{R}}$ are obtained by solving the equation $\operatorname{det}\left(\lambda_{k} \mathbf{I}-\widehat{\mathbf{R}}\right)=0$. For $3 \times 3$ symmetric matrix, the analytic solutions are given by (see [29])

$$
\lambda_{k}=\frac{2 \sqrt{p}}{3} \cos \left(\varphi+\frac{2 k \pi}{3}\right)-\frac{1}{3} c_{2}, k=0,1,2
$$




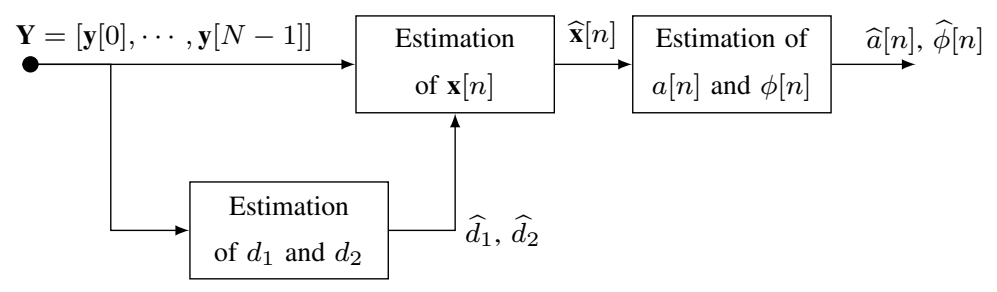

Fig. 1: Estimation procedure for $a[n], \phi[n], d_{1}$ and $d_{2}$.

where

$$
\begin{aligned}
\varphi & =\frac{1}{3} \arctan \left(\frac{\left.\sqrt{27\left[\frac{1}{4} c_{1}^{2}\left(p-c_{1}\right)+c_{0}\left(q+\frac{27}{4} c_{0}\right)\right.}\right]}{q}\right) \\
c_{2} & =-r_{00}-r_{11}-r_{22} \\
c_{1} & =r_{00} r_{11}+r_{00} r_{22}+r_{11} r_{22}-r_{01}^{2}-r_{02}^{2}-r_{12}^{2} \\
c_{0} & =r_{00} r_{12}^{2}+r_{11} r_{02}^{2}+r_{22} r_{01}^{2}-r_{00} r_{11} r_{22}-2 r_{02} r_{01} r_{12} \\
p & =c_{2}^{2}-3 c_{1} \\
q & =-\frac{27}{2} c_{0}-c_{2}^{3}+\frac{9}{2} c_{2} c_{1} .
\end{aligned}
$$

In particular, as $\varphi \in\left[0, \frac{2 \pi}{3}\right.$ [, we can easily show that the smallest eigenvalue is given by $\lambda_{\min }=\lambda_{1}$. The associated eigenvector, $\widehat{\mathbf{u}}$, is obtained by solving the equation $\widehat{\mathbf{R}} \widehat{\mathbf{u}}=\lambda_{\min } \widehat{\mathbf{u}}$. After some computations, we obtain

$$
\begin{aligned}
& \widehat{u}_{0}=\beta \times\left(\left(r_{22}-\lambda_{\min }\right)\left(r_{11}-\lambda_{\min }\right)-r_{12}^{2}\right) \\
& \widehat{u}_{1}=\beta \times\left(r_{02} r_{12}-r_{01}\left(r_{22}-\lambda_{\min }\right)\right) \\
& \widehat{u}_{2}=\beta \times\left(r_{01} r_{12}-r_{02}\left(r_{11}-\lambda_{\min }\right)\right)
\end{aligned}
$$

where the normalized coefficient $\beta$ is introduced to enforce the constraint $\|\widehat{\mathbf{u}}\|_{F}^{2}=1$. Finally, inserting (34) into (25) yields

$$
\begin{aligned}
& \widehat{d}_{1}=\frac{\left(r_{22}-\lambda_{\min }\right)\left(r_{11}-\lambda_{\min }\right)-r_{12}^{2}}{r_{02} r_{12}-r_{01}\left(r_{22}-\lambda_{\min }\right)} \\
& \widehat{d}_{2}=\frac{\left(r_{22}-\lambda_{\min }\right)\left(r_{11}-\lambda_{\min }\right)-r_{12}^{2}}{r_{01} r_{12}-r_{02}\left(r_{11}-\lambda_{\min }\right)}
\end{aligned}
$$




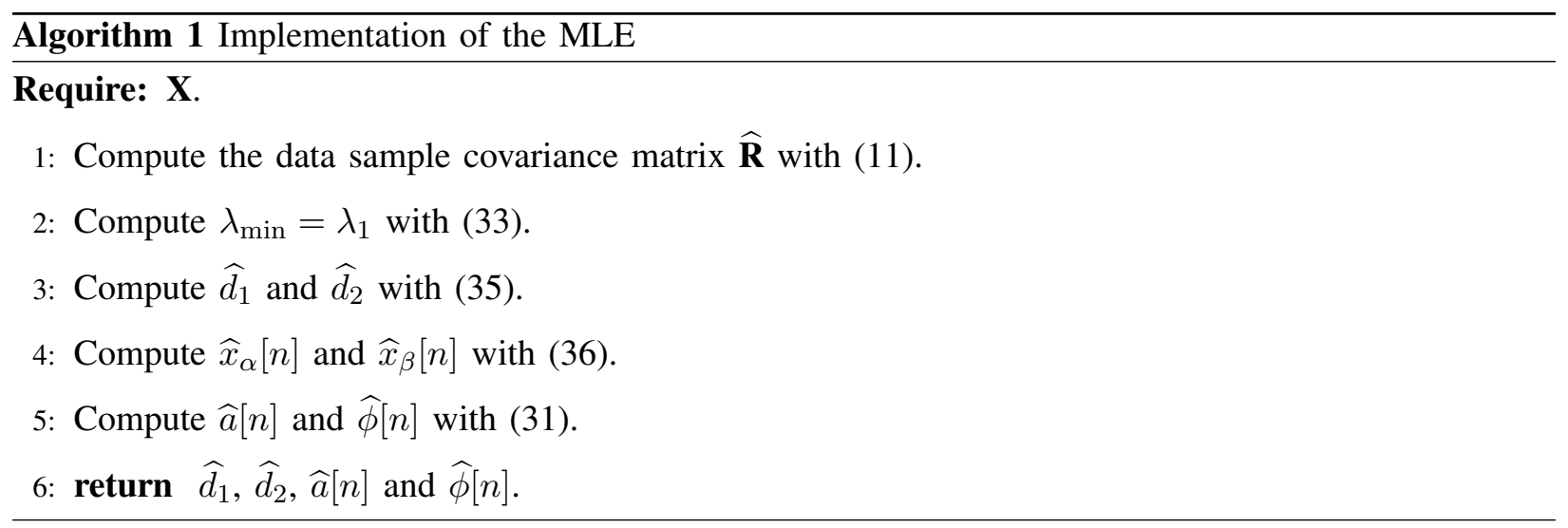

2) Expression of $x_{\alpha}[n]$ and $x_{\beta}[n]$ : The estimate of $\mathbf{x}[n]=\left[\widehat{x}_{0}[n], \widehat{x}_{1}[n]\right]^{T}$ is given by (28). This estimate can be expressed in a simple form using the value of $\left(\mathbf{H}^{T} \widehat{\mathbf{D}}^{2} \mathbf{H}\right)^{-1}$ in (14). After some computations, we find

$$
\begin{aligned}
& \widehat{x}_{\alpha}[n]=\frac{\left(\widehat{d}_{1}^{2}+\widehat{d}_{2}^{2}\right) y_{0}[n]-\widehat{d}_{1} \widehat{d}_{2}^{2} y_{1}[n]-\widehat{d}_{1}^{2} \widehat{d}_{2} y_{2}[n]}{\widehat{d}_{2}^{2}+\widehat{d}_{1}^{2}+\widehat{d}_{1}^{2} \widehat{d}_{2}^{2}} \\
& \widehat{x}_{\beta}[n]=\frac{\left(\widehat{d}_{1}^{2}-\widehat{d}_{2}^{2}\right) y_{0}[n]+\widehat{d}_{1}\left(\widehat{d}_{2}^{2}+2\right) y_{1}[n]-\widehat{d}_{2}\left(\widehat{d}_{1}^{2}+2\right) y_{2}[n]}{\sqrt{3}\left(\widehat{d}_{2}^{2}+\widehat{d}_{1}^{2}+\widehat{d}_{1}^{2} \widehat{d}_{2}^{2}\right)}
\end{aligned}
$$

Finally, the implementation is detailed in Algorithm 1. For the computation of $\widehat{\phi}[n]$ in (31) and $\varphi$ in (33), care must be taken to resolve the ambiguity in the arctan function since $\phi[n] \in[02 \pi[$ and $\varphi \in\left[\begin{array}{ll}0 & 2 \pi / 3\end{array}\right.$. In practice, the correct quadrant can be obtained directly using the atan2 function which is provided in the standard library of most programming languages. We should emphasize that the proposed technique is easy to implement and does not require any external library. Therefore, our solution makes it also a potential (if not the unique) candidate for implementation on hardware embedded system (e.g. FPGA, microprocessors).

\section{Performance Analysis: Cramér Rao Bounds}

A natural criterion to assess the performance of an estimator, $\theta$, is the mean square error (MSE). The MSE can be decomposed as follows [28]

$$
\operatorname{MSE}[\widehat{\theta}]=\operatorname{bias}^{2}(\theta)+\operatorname{var}(\widehat{\theta})
$$

where $\operatorname{bias}(\theta)$ and $\operatorname{var}(\widehat{\theta})$ correspond to the bias and variance of the estimator, respectively. For unbiased estimators, the variance is lower bounded by the so-called Cramér-Rao Lower Bound (CRB). In this context, an unbiased estimator that reaches the CRB is said to be efficient [28]. 
The statistical performance of the Maximum Likelihood Estimator under the Conditional model (CML) has been characterized in several studies. Specifically, it has been demonstrated in [30], [31] that the CML is asymptotically unbiased as $N \rightarrow \infty$ or $\mathrm{SNR} \rightarrow \infty$, making the comparison between the variance and $\mathrm{CRB}$ relevant in the asymptotic regions. Furthermore, it has also been demonstrated that the variance of the CML reaches the CRB at high SNR [31], a property which is not systematically shared by other estimators $^{2}$. Finally, it has been shown that the CML does not reach the CRB for $N \rightarrow \infty$ (with $\operatorname{SNR} \neq \infty$ ). In particular, the non-efficiency of the CML for $N \rightarrow \infty$ is due to the fact that the number of unknowns grows without bound as $N$ increases $^{3}$ [24], [33].

This section focuses on the derivation of the Cramér-Rao Lower Bound for the Conditional Model in (2). It should be mentioned that the CRB of the fundamental frequency has been empirically analysed in [34] for unbalanced three-phase systems with constant IA and linear IP. In this section, we provide explicit expressions for the CRBs of the unbalance parameters, IA and IP for the general case, and we highlight some useful properties of these bounds.

The Cramér-Rao inequality states that the variance of any unbiased estimator is bounded by

$$
\begin{aligned}
\operatorname{var}(\widehat{a}[n]) & \geq \mathrm{CRB}[a[n]] \\
\operatorname{var}(\widehat{\phi}[n]) & \geq \mathrm{CRB}[\phi[n]] \\
\operatorname{var}\left(\widehat{d}_{k}[n]\right) & \geq \mathrm{CRB}\left[d_{k}\right]
\end{aligned}
$$

where $\widehat{a}[n], \widehat{\phi}[n]$ and $\widehat{d}_{k}[n]$ correspond to the estimates of $a[n], \phi[n]$ and $d_{k}$, respectively. To derive explicit expressions for the CRBs, let us define

$$
\mathbf{T}(\Omega) \triangleq\left[\begin{array}{c}
\operatorname{DHG}(a[0], \phi[0]) \\
\operatorname{DHG}(a[1], \phi[1]) \\
\vdots \\
\mathbf{D H G}(a[N-2], \phi[N-2]) \\
\mathbf{D H G}(a[N-1], \phi[N-1])
\end{array}\right]
$$

the $3 N \times 1$ column vector containing the noiseless samples.

The CRBs are derived from the inverse of the Fisher Information Matrix. As $\mathbf{b}[n] \sim \mathcal{N}\left(0, \sigma^{2} \mathbf{I}\right)$, the

${ }^{2}$ For example, the Unconditional Maximum Likelihood does not reach the CRB at finite number of samples and high signal to noise ratio [32].

${ }^{3}$ In our context, the number of unknowns is equal to $2(N+1)$. 
$i j^{\text {th }}$ element of the Fisher Information Matrix is given by [28]

$$
[\mathbf{F}(\Omega)]_{i, j}=\frac{1}{\sigma^{2}}\left(\frac{\partial \mathbf{T}(\Omega)}{\partial \Omega_{i}}\right)^{T}\left(\frac{\partial \mathbf{T}(\Omega)}{\partial \Omega_{j}}\right)
$$

where $\Omega_{j}$ corresponds to the $(j+1)^{t h}$ element of $\Omega$ (see (7)). From the definition of $\mathbf{T}(\Omega)$, it follows that

$$
\begin{aligned}
{[\mathbf{F}(\Omega)]_{i, j} } & =\frac{1}{\sigma^{2}} \sum_{n=0}^{N-1}\left(\frac{\partial \mathbf{D H} \mathbf{H}[n]}{\partial \Omega_{i}}\right)^{T}\left(\frac{\partial \mathbf{D H} \mathbf{x}[n]}{\partial \Omega_{j}}\right) \\
& =[\mathbf{F}(\Omega)]_{j, i}
\end{aligned}
$$

After some computations, we find

$$
\mathbf{F}(\Omega)=\left[\begin{array}{ccccc}
\mathbf{K}_{0} & \mathbf{0} & \cdots & \mathbf{0} & \mathbf{Z}_{0}^{T} \\
\mathbf{0} & \mathbf{K}_{1} & \ddots & \vdots & \mathbf{Z}_{1}^{T} \\
\vdots & \ddots & \ddots & \mathbf{0} & \vdots \\
\mathbf{0} & \cdots & \mathbf{0} & \mathbf{K}_{N-1} & \mathbf{Z}_{N-1}^{T} \\
\mathbf{Z}_{0} & \mathbf{Z}_{1} & \cdots & \mathbf{Z}_{N-1} & \boldsymbol{\Gamma}
\end{array}\right]
$$

where

$$
\begin{aligned}
\mathbf{K}_{n} & =\frac{1}{\sigma^{2}} \mathbf{Q}[n] \mathbf{H}^{T} \mathbf{D}^{2} \mathbf{H} \mathbf{Q}^{T}[n] \\
\mathbf{Z}_{n} & =\frac{1}{\sigma^{2}}\left[\begin{array}{c}
d_{1} \mathbf{x}^{T}[n] \mathbf{h}_{1}^{T} \mathbf{h}_{1} \\
d_{2} \mathbf{x}^{T}[n] \mathbf{h}_{2}^{T} \mathbf{h}_{2}
\end{array}\right] \mathbf{Q}^{T}[n] \\
\boldsymbol{\Gamma} & =\frac{N}{\sigma^{2}}\left[\begin{array}{cc}
\mathbf{h}_{1} \mathbf{R}_{\mathbf{x}} \mathbf{h}_{1} & 0 \\
0 & \mathbf{h}_{2} \mathbf{R}_{\mathbf{x}} \mathbf{h}_{2}
\end{array}\right]
\end{aligned}
$$

and

$$
\begin{aligned}
& \mathbf{Q}[n] \triangleq\left[\begin{array}{cc}
\cos (\phi[n]) & \sin (\phi[n]) \\
-a[n] \sin (\phi[n]) & a[n] \cos (\phi[n])
\end{array}\right] \\
& \mathbf{h}_{1} \triangleq\left[-\frac{1}{2} \frac{\sqrt{3}}{2}\right] \\
& \mathbf{h}_{2} \triangleq\left[-\frac{1}{2}-\frac{\sqrt{3}}{2}\right] \\
& \mathbf{R}_{\mathbf{x}} \triangleq \frac{1}{N} \sum_{n=0}^{N-1} \mathbf{x}[n] \mathbf{x}^{T}[n]
\end{aligned}
$$

Let us decompose the Fisher Information Matrix as

$$
\mathbf{F}(\Omega)=\left[\begin{array}{cc}
\mathbf{K} & \mathbf{Z}^{T} \\
\mathbf{Z} & \boldsymbol{\Gamma}
\end{array}\right]
$$


where

- $\mathbf{K}$ is a $2 N \times 2 N$ matrix which is defined as

$$
\mathbf{K} \triangleq\left[\begin{array}{ccc}
\mathbf{K}_{0} & \mathbf{0} & \mathbf{0} \\
\mathbf{0} & \ddots & \mathbf{0} \\
\mathbf{0} & \mathbf{0} & \mathbf{K}_{N-1}
\end{array}\right]
$$

- and $\mathbf{Z}$ is a $2 \times 2 N$ matrix which is defined as

$$
\mathbf{Z} \triangleq\left[\mathbf{Z}_{0}, \ldots, \mathbf{Z}_{N-1}\right]
$$

Using the inverse formula for partitioned matrix [28], we obtain

$$
\begin{aligned}
& \mathbf{F}^{-1}(\Omega)= \\
& {\left[\begin{array}{cr}
\left(\mathbf{K}-\mathbf{Z}^{T} \boldsymbol{\Gamma}^{-1} \mathbf{Z}\right)^{-1} & -\left(\mathbf{K}-\mathbf{Z}^{T} \boldsymbol{\Gamma}^{-1} \mathbf{Z}\right)^{-1} \mathbf{Z}^{T} \boldsymbol{\Gamma}^{-1} \\
-\left(\boldsymbol{\Gamma}-\mathbf{Z} \mathbf{K}^{-1} \mathbf{Z}^{T}\right)^{-1} \mathbf{Z} \mathbf{K}^{-1} & \left(\boldsymbol{\Gamma}-\mathbf{Z} \mathbf{K}^{-1} \mathbf{Z}^{T}\right)^{-1}
\end{array}\right]}
\end{aligned}
$$

Using (42c) and (45), it can be checked that the inverses $\boldsymbol{\Gamma}^{-1}$ and $\mathbf{K}^{-1}$ are equal to

$$
\begin{aligned}
\boldsymbol{\Gamma}^{-1} & =\frac{\sigma^{2}}{N}\left[\begin{array}{cc}
\frac{1}{\mathbf{h}_{1} \mathbf{R}_{\mathbf{x}} \mathbf{h}_{1}^{T}} & 0 \\
0 & \frac{1}{\mathbf{h}_{2} \mathbf{R}_{\mathbf{x}} \mathbf{h}_{2}^{T}}
\end{array}\right] \\
\mathbf{K}^{-1} & =\left[\begin{array}{ccc}
\mathbf{K}_{0}^{-1} & \mathbf{0} & \mathbf{0} \\
\mathbf{0} & \ddots & \mathbf{0} \\
\mathbf{0} & \mathbf{0} & \mathbf{K}_{N-1}^{-1}
\end{array}\right]
\end{aligned}
$$

where $\mathbf{K}_{n}^{-1}$ is equal to

$$
\mathbf{K}_{n}^{-1}=\sigma^{2} \mathbf{Q}^{-T}[n]\left(\mathbf{H}^{T} \mathbf{D}^{2} \mathbf{H}\right)^{-1} \mathbf{Q}^{-1}[n]
$$

\section{A. CRBs of the Unbalance Parameters}

The Cramér-Rao Bounds of $d_{1}$ and $d_{2}$ are defined by

$$
\begin{aligned}
& \mathrm{CRB}\left[d_{1}\right] \triangleq\left[\mathbf{F}^{-1}(\Omega)\right]_{2 N, 2 N} \\
& \mathrm{CRB}\left[d_{2}\right] \triangleq\left[\mathbf{F}^{-1}(\Omega)\right]_{2 N+1,2 N+1}
\end{aligned}
$$

The computation of the Cramér-Rao Bounds requires the inversion of $\boldsymbol{\Gamma}-\mathbf{Z} \mathbf{K}^{-1} \mathbf{Z}^{T}$, which can be expressed in a simple form.

From (49), (48), (46) and (42b), it can be verified that

$$
\mathbf{Z K}^{-1} \mathbf{Z}^{T}=\frac{N}{\sigma^{2}}\left[\begin{array}{cc}
d_{1}^{2} p_{11} \mathbf{h}_{1} \mathbf{R}_{\mathbf{x}} \mathbf{h}_{1}^{T} & d_{1} d_{2} p_{12} \mathbf{h}_{2} \mathbf{R}_{\mathbf{x}} \mathbf{h}_{1}^{T} \\
d_{1} d_{2} p_{12} \mathbf{h}_{2} \mathbf{R}_{\mathbf{x}} \mathbf{h}_{1}^{T} & d_{2}^{2} p_{11} \mathbf{h}_{2} \mathbf{R}_{\mathbf{x}} \mathbf{h}_{2}^{T}
\end{array}\right]
$$


where $p_{u v} \triangleq \mathbf{h}_{u}\left(\mathbf{H}^{T} \mathbf{D H}\right)^{-1} \mathbf{h}_{v}^{T}$. Specifically, using (14), we find

$$
p_{11}=\frac{d_{2}^{2}+1}{\|\mathbf{v}(\mathbf{d})\|^{2}}, \quad p_{12}=\frac{-1}{\|\mathbf{v}(\mathbf{d})\|^{2}}, \quad p_{22}=\frac{d_{1}^{2}+1}{\|\mathbf{v}(\mathbf{d})\|^{2}},
$$

where $\mathbf{v}(\mathbf{d})$ is defined in (18). Then, using (42c), (51) and (52), it follows that

$$
\boldsymbol{\Gamma}-\mathbf{Z K}^{-1} \mathbf{Z}^{T}=\frac{N}{\sigma^{2}\|\mathbf{v}(\mathbf{d})\|^{2}}\left[\begin{array}{cc}
d_{2}^{2} \mathbf{h}_{1} \mathbf{R}_{\mathbf{x}} \mathbf{h}_{1}^{T} & d_{1} d_{2} \mathbf{h}_{2} \mathbf{R}_{\mathbf{x}} \mathbf{h}_{1}^{T} \\
d_{1} d_{2} \mathbf{h}_{2} \mathbf{R}_{\mathbf{x}} \mathbf{h}_{1}^{T} & d_{1}^{2} \mathbf{h}_{2} \mathbf{R}_{\mathbf{x}} \mathbf{h}_{2}^{T}
\end{array}\right]
$$

Finally, the CRBs are obtained by inverting $\Gamma-\mathbf{Z} \mathbf{K}^{-1} \mathbf{Z}^{T}$. As $\mathbf{h}_{1} \mathbf{R}_{\mathbf{x}} \mathbf{h}_{1}^{T} \mathbf{h}_{2} \mathbf{R}_{\mathbf{x}} \mathbf{h}_{2}^{T}-\mathbf{h}_{2} \mathbf{R}_{\mathbf{x}} \mathbf{h}_{1}^{T} \mathbf{h}_{2} \mathbf{R}_{\mathbf{x}} \mathbf{h}_{1}^{T}=$ $(3 / 4) \operatorname{det}\left(\mathbf{R}_{\mathbf{x}}\right)$, we obtain

$$
\begin{aligned}
& \mathrm{CRB}\left[d_{1}\right]=\frac{4 \sigma^{2}\|\mathbf{v}(\mathbf{d})\|^{2}}{3 N} \times \frac{\mathbf{h}_{2} \mathbf{R}_{\mathbf{x}} \mathbf{h}_{2}^{T}}{d_{2}^{2} \operatorname{det}\left(\mathbf{R}_{\mathbf{x}}\right)} \\
& \mathrm{CRB}\left[d_{2}\right]=\frac{4 \sigma^{2}\|\mathbf{v}(\mathbf{d})\|^{2}}{3 N} \times \frac{\mathbf{h}_{1} \mathbf{R}_{\mathbf{x}} \mathbf{h}_{1}^{T}}{d_{1}^{2} \operatorname{det}\left(\mathbf{R}_{\mathbf{x}}\right)} .
\end{aligned}
$$

We observe that $\mathrm{CRB}\left[d_{1}\right]$ and $\mathrm{CRB}\left[d_{2}\right]$ tend to 0 when $N \rightarrow \infty$ or when $\sigma^{2} \rightarrow 0$. Additional properties are also provided in Remark 2 and 3.

Remark 2: The CRBs of $d_{1}$ and $d_{2}$ are bounded by

$$
\begin{aligned}
& \frac{4 \sigma^{2}\|\mathbf{v}(\mathbf{d})\|^{2}}{3 N l_{\max } d_{2}^{2}} \leq \mathrm{CRB}\left[d_{1}\right] \leq \frac{4 \sigma^{2}\|\mathbf{v}(\mathbf{d})\|^{2}}{3 N l_{\min } d_{2}^{2}} \\
& \frac{4 \sigma^{2}\|\mathbf{v}(\mathbf{d})\|^{2}}{3 N l_{\max } d_{1}^{2}} \leq \mathrm{CRB}\left[d_{2}\right] \leq \frac{4 \sigma^{2}\|\mathbf{v}(\mathbf{d})\|^{2}}{3 N l_{\min } d_{1}^{2}} .
\end{aligned}
$$

where $l_{\max } \geq l_{\min }$ are the eigenvalues of $\mathbf{R}_{\mathbf{x}}$.

Proof: First, as $\mathbf{h}_{u} \mathbf{h}_{u}^{T}=1$, the scalar $\mathbf{h}_{u} \mathbf{R}_{\mathbf{x}} \mathbf{h}_{u}^{T}$ is bounded by $l_{\min } \leq \mathbf{h}_{u} \mathbf{R}_{\mathbf{x}} \mathbf{h}_{u}^{T} \leq l_{\max }$. Then, as the matrix determinant is equal to the product of the eigenvalues of $\mathbf{R}_{\mathbf{x}}, \operatorname{det}\left(\mathbf{R}_{\mathbf{x}}\right)=l_{\min } l_{\max }$. Using these two properties in (54) yields to (55).

Remark 3: When $\mathbf{R}_{\mathbf{x}}=\sigma_{s}^{2} \mathbf{I}$, the CRBs of $d_{1}$ and $d_{2}$ are related by

$$
\frac{\mathrm{CRB}\left[d_{2}\right]}{\mathrm{CRB}\left[d_{1}\right]}=\left(\frac{d_{2}}{d_{1}}\right)^{2}
$$

Proof: The proof comes from the fact that $\mathbf{h}_{1} \mathbf{h}_{1}^{T}=1$ and $\mathbf{h}_{2} \mathbf{h}_{2}^{T}=1$.

\section{B. CRBs of the Instantaneous Amplitude and Instantaneous Phase}

The Cramér-Rao Bounds of $a[n]$ and $\phi[n]$ are defined by

$$
\begin{aligned}
\mathrm{CRB}[a[n]] & \triangleq\left[\mathbf{F}^{-1}(\Omega)\right]_{2 n, 2 n} \\
\mathrm{CRB}[\phi[n]] & \triangleq\left[\mathbf{F}^{-1}(\Omega)\right]_{2 n+1,2 n+1}
\end{aligned}
$$


The computation of the Cramér-Rao Bounds requires the inversion of $\mathbf{K}-\mathbf{Z}^{T} \boldsymbol{\Gamma}^{-1} \mathbf{Z}$, which is difficult to obtain in closed-form. However, this inverse matrix can be approximated for the asymptotic case $N \rightarrow \infty$. Indeed, when $\mathbf{R}_{\mathbf{x}}$ is finite, we obtain the following limit

$$
\mathbf{Z}^{T} \boldsymbol{\Gamma}^{-1} \mathbf{Z}=\frac{\sigma^{2}}{N} \mathbf{Z}^{T}\left[\begin{array}{cc}
\frac{1}{\mathbf{h}_{1} \mathbf{R}_{\mathbf{x}} \mathbf{h}_{1}^{T}} & 0 \\
0 & \frac{1}{\mathbf{h}_{2} \mathbf{R}_{\mathbf{x}} \mathbf{h}_{2}^{T}}
\end{array}\right] \mathbf{Z} \rightarrow \mathbf{0}
$$

Therefore, it follows that

$$
\left(\mathbf{K}-\mathbf{Z}^{T} \boldsymbol{\Gamma}^{-1} \mathbf{Z}\right)^{-1} \rightarrow \mathbf{K}^{-1}
$$

For the asymptotic case, this approximation shows that the contribution of the estimation error of $d_{1}$ and $d_{2}$ can be neglected. From (48), we obtain the asymptotic Cramér-Rao Bounds of $a[n]$ and $\phi[n]$. These bounds are equal to

$$
\begin{aligned}
\mathrm{CRB}_{\infty}[a[n]] & =\left[\mathbf{K}_{n}^{-1}\right]_{0,0} \\
\mathrm{CRB}_{\infty}[\phi[n]] & =\left[\mathbf{K}_{n}^{-1}\right]_{1,1}
\end{aligned}
$$

In order to obtain a closed-form expression, we make use of the following equality (see the definition of $\mathbf{Q}[n]$ in (43a))

$$
\mathbf{Q}^{-1}[n]=\left[\begin{array}{cc}
\cos (\phi[n]) & -\frac{1}{a[n]} \sin (\phi[n]) \\
\sin (\phi[n]) & \frac{1}{a[n]} \cos (\phi[n])
\end{array}\right]
$$

Using this equality in (49) yields to

$$
\begin{aligned}
\mathrm{CRB}_{\infty}[a[n]] & =\sigma^{2} \mathbf{q}_{1}[n]\left(\mathbf{H}^{T} \mathbf{D}^{2} \mathbf{H}\right)^{-1} \mathbf{q}_{1}^{T}[n] \\
\mathrm{CRB}_{\infty}[\phi[n]] & =\frac{\sigma^{2}}{a^{2}[n]} \mathbf{q}_{2}[n]\left(\mathbf{H}^{T} \mathbf{D}^{2} \mathbf{H}\right)^{-1} \mathbf{q}_{2}^{T}[n]
\end{aligned}
$$

where

$$
\begin{aligned}
& \mathbf{q}_{1}[n] \triangleq[\cos (\phi[n]) \sin (\phi[n])] \\
& \mathbf{q}_{2}[n] \triangleq[-\sin (\phi[n]) \cos (\phi[n])]
\end{aligned}
$$

From (60), we see that the asymptotic Cramér Rao Bounds do not depend on $N$ and increase linearly with the noise variance $\sigma^{2}$. In particular, these bounds tend to 0 only if $\sigma^{2} \rightarrow 0$. We also observe that $\mathrm{CRB}_{\infty}[a[n]]$ does not depend on $a[n]$, while $\mathrm{CRB}_{\infty}[\phi[n]]$ decreases quadratically with $a[n]$. When $a[n]=0$, it is interesting to note that the estimation of $\phi[n]$ becomes impossible. Finally, we observe that the two CRBs depend on $\phi[n]$, and their derivatives are related by

$$
\frac{\partial \mathrm{CRB}_{\infty}(a[n])}{\partial \phi[n]}=-a[n]^{2}\left(\frac{\partial \mathrm{CRB}_{\infty}(\phi[n])}{\partial \phi[n]}\right)
$$


which implies that the positions of the extrema are the same but their natures (minima or maxima) are different.

\section{NUMERICAL EXAMPLES}

In this section, the performances of the proposed technique are evaluated using synthetic signals as defined in the IEEE Standard C37.118 [9]. The accuracy of the proposed estimators is assessed with the following Mean Square Errors (MSEs)

$$
\begin{aligned}
& \operatorname{MSE}\left[d_{k}\right]=E\left[\left(\widehat{d}_{k}-d_{k}\right)^{2}\right] \\
& \operatorname{MSE}[a]=\frac{1}{N} \sum_{n=0}^{N-1} E\left[(\widehat{a}[n]-a[n])^{2}\right] \\
& \operatorname{MSE}[\phi]=\frac{1}{N} \sum_{n=0}^{N-1} E\left[[\widehat{\phi}[n]-\phi[n]]_{[-\pi, \pi(}^{2}\right]
\end{aligned}
$$

where the notation $[.]_{[-\pi, \pi(}$ in (63c) means that the phase error is reduced to the interval $[-\pi, \pi($, and $E[]$. corresponds to the expectation operator. In the following, the expectations are estimated through 5000 Monte Carlo trials. As the MLE is asymptotically unbiased, we compare the MSEs of the unbalance parameters to the Cramér-Rao Bounds $\mathrm{CRB}\left[d_{1}\right]$ and $\mathrm{CRB}\left[d_{2}\right]$, and the MSEs of the IA and IP to the average CRBs

$$
\begin{aligned}
\operatorname{CRB}[a] & =\frac{1}{N} \sum_{n=0}^{N-1} \operatorname{CRB}[a[n]] \\
\mathrm{CRB}[\phi] & =\frac{1}{N} \sum_{n=0}^{N-1} \mathrm{CRB}[\phi[n]] .
\end{aligned}
$$

In order to show the accuracy of the CRB approximation in (60), $\mathrm{CRB}[a]$ and $\mathrm{CRB}[\phi]$ are also compared with those obtained with the asymptotic CRBs.

In the following experiments, the unbalance parameters are set to $d_{0}=1, d_{1}=0.75, d_{2}=1.199^{4}$, the nominal frequency is fixed to $f_{s}=60 \mathrm{~Hz}$, and the sampling frequency is equal to $F_{s}=5 \mathrm{kHz}$.

\footnotetext{
${ }^{4}$ The values of $d_{k}$ have been chosen to respect both the normalization of the proposed technique, i.e. $d_{0}=1$, and the one of the PCA approach, i.e. $d_{0}^{2}+d_{1}^{2}+d_{2}^{2}=3$.
} 


\begin{tabular}{|c|cc|ccc|ccc|}
\cline { 3 - 8 } \multicolumn{2}{c|}{} & \multicolumn{3}{c|}{$\begin{array}{c}\text { Experimental } \\
\text { values }\end{array}$} & \multicolumn{3}{c|}{$\begin{array}{c}\text { Approximated values } \\
\text { using }(26)\end{array}$} \\
\hline \multirow{5}{*}{\multicolumn{1}{c|}{}} & $N$ & CRB & MSE & $\operatorname{var}\left(\widehat{d}_{k}\right)$ & biais $^{2}\left(d_{k}\right)$ & MSE & var $\left(\widehat{d}_{k}\right)$ & biais $^{2}\left(d_{k}\right)$ \\
& samples & $\times 10^{-4}$ & $\times 10^{-4}$ & $\times 10^{-4}$ & $\times 10^{-4}$ & $\times 10^{-4}$ & $\times 10^{-4}$ & $\times 10^{-4}$ \\
\hline \multirow{4}{*}{$d_{1}$} & 120 & 18.0 & 19.3 & 19.3 & 0.0 & 20.1 & 20.0 & 0.0 \\
& 200 & 10.8 & 11.6 & 11.6 & 0.0 & 12.0 & 12.0 & 0.0 \\
& 1000 & 2.1 & 2.2 & 2.2 & 0.0 & 2.4 & 2.4 & 0.0 \\
\hline \multirow{4}{*}{$d_{2}$} & 120 & 44.6 & 46.5 & 46.5 & 0.0 & 48.6 & 48.6 & 0.0 \\
& 200 & 26.5 & 27.9 & 27.9 & 0.0 & 29.2 & 29.1 & 0.0 \\
& 1000 & 5.3 & 5.7 & 5.7 & 0.0 & 5.8 & 5.8 & 0.0 \\
\hline
\end{tabular}

TABLE I: Linear FM: CRB, variance and square of the bias of the estimators $d_{k}$ versus data length $\left(\sigma^{2}=4 \cdot 10^{-2}\right)$.

\section{A. Estimation with Linear Frequency Modulation (FM)}

The first test investigates the case of three-phase signals with unit amplitude and linear frequency modulation. Under these conditions, the IA and IP are respectively given by [9, 5.5.7]

$$
\begin{aligned}
& a[n]=1 \\
& \phi[n]=2 \pi f_{s} n / F_{s}+\pi r_{f}\left(n / F_{s}\right)^{2}
\end{aligned}
$$

where $r_{f}$ is the frequency ramp rate, which is set to $r_{f}=1 \mathrm{~Hz} / \mathrm{s}[9,5.5 .7]$. The corresponding signal is displayed in Figure 2a for illustration.

Statistical performances are analysed for different data lengths, and at different signal-to-noise ratios (SNRs). The SNR is defined

$$
\mathbf{S N R}_{d B}=10 \log \left(\frac{\operatorname{Tr}\left[\mathbf{D H} \mathbf{R}_{\mathbf{x}} \mathbf{H}^{T} \mathbf{D}^{T}\right]}{3 \sigma^{2}}\right) .
$$

Tables I and II present the CRB, MSE, variance and (the square of) the bias of $d_{k}(k=1,2)$ versus the data length and SNR, respectively. The approximated values of the variance and bias in (26) are also provided for comparison. First, we observe that the CRB, MSE, variance and bias decrease as the data length or SNR increases. Then, we note that the square of the bias is close or equal to zero in each simulation, making the comparison between the CRB and MSE relevant in the asymptotic regions. By comparing the CRB and MSE in Table I, we show that the MSEs do not achieve the CRBs at fixed SNR since the difference between the CRB and MSEs is non-zero, even with a large number of samples. This behaviour is not observed in Table II. These observations corroborate the fact that the CML is not 


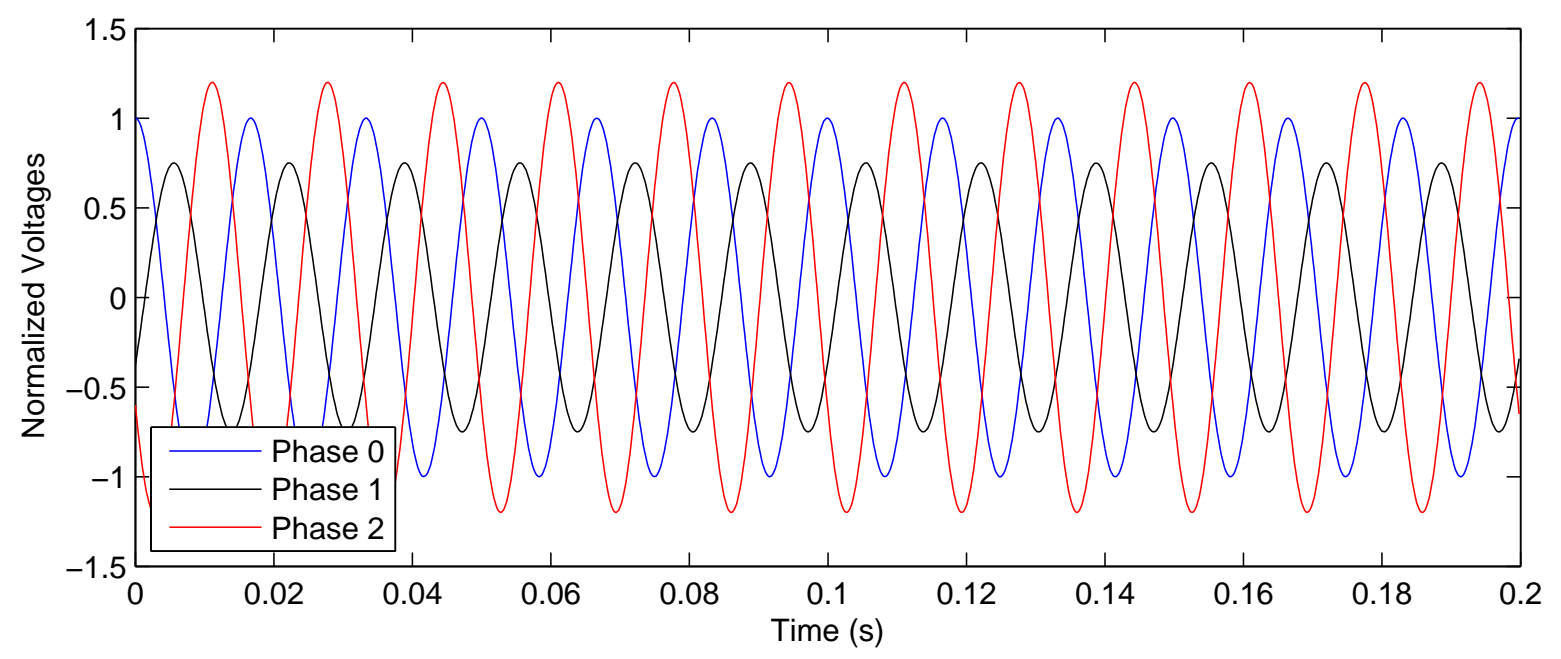

(a) Linear frequency modulation $\left(r_{f}=1 \mathrm{~Hz} / \mathrm{s}\right)$

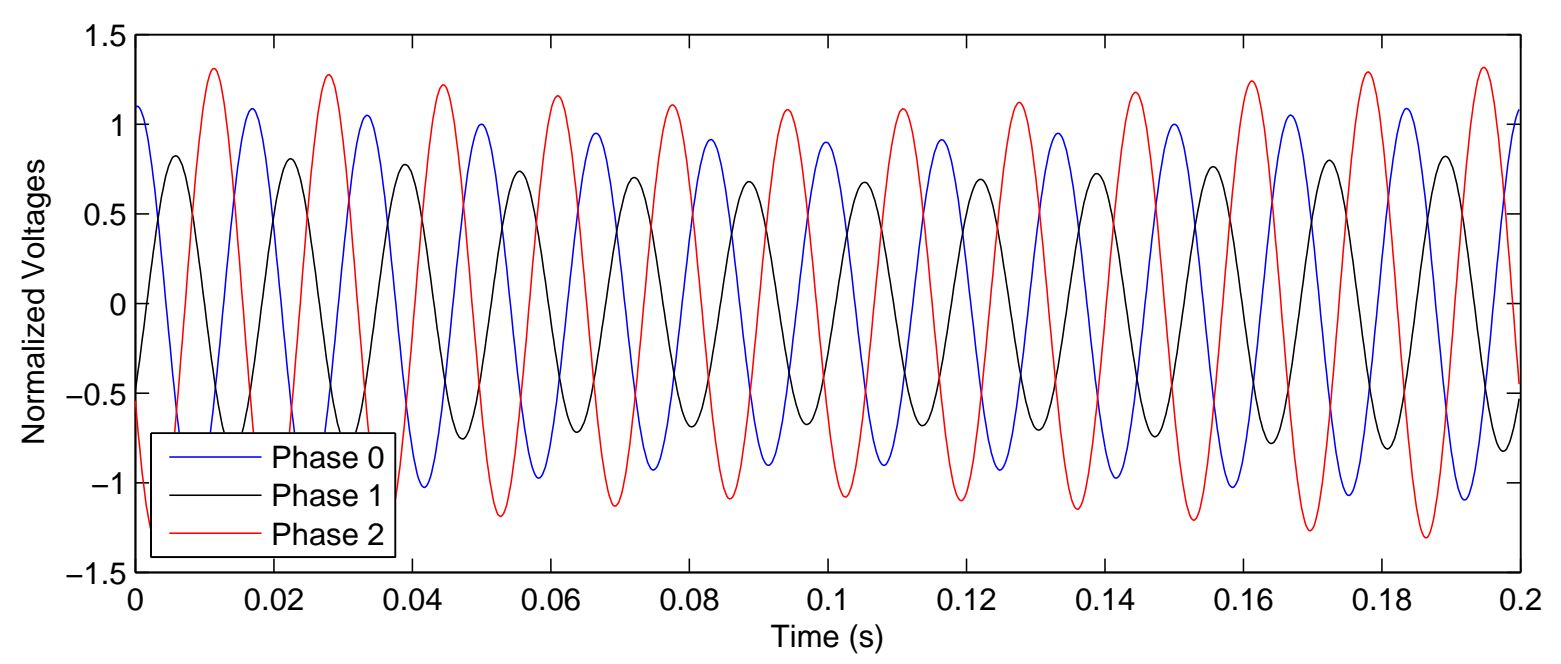

(b) Sinusoidal amplitude and phase modulation $\left(k_{x}=k_{a}=0.1\right.$ and $\left.f_{m}=5 \mathrm{~Hz}\right)$

Fig. 2: Three-phase signals according to the IEEE Standard C37.118.1 with amplitude unbalance $\left(d_{1}=\right.$ $0.75, d_{2}=1.199$ and $\left.\sigma^{2}=0\right)$

efficient for large $N$ (at fixed SNR) [24], [30], [33], and efficient for large SNR (at fixed $N$ ) [31]. In particular, as discussed in section IV, the non-efficiency of the Maximum Likelihood for $N \rightarrow \infty$ is due to the fact that the number of unknown parameters grows without bound as $N$ increases [30]. Finally, we note that the approximated values of the MSE, variance and bias are close to the experimental ones. However, it should be mentioned that a small error still persists even for large $N$. For example, with $N=1000$ samples, the experimental MSE of $d_{1}$ is equal to $2.2 \times 10^{-4}$ whereas the approximated MSE 


\begin{tabular}{|c|cc|ccc|ccc|}
\cline { 3 - 8 } \multicolumn{2}{c|}{} & \multicolumn{3}{c|}{$\begin{array}{c}\text { Experimental } \\
\text { values }\end{array}$} & \multicolumn{3}{c|}{ Approximated values } \\
using $(26)$ \\
\hline \multirow{5}{*}{$d_{1}$} & $S N R$ & CRB & MSE & $\operatorname{var}\left(\widehat{d}_{k}\right)$ & biais $^{2}\left(d_{k}\right)$ & MSE & $\operatorname{var}\left(\widehat{d}_{k}\right)$ & biais $^{2}\left(d_{k}\right)$ \\
& $\mathrm{dB}$ & $\times 10^{-4}$ & $\times 10^{-4}$ & $\times 10^{-4}$ & $\times 10^{-4}$ & $\times 10^{-4}$ & $\times 10^{-4}$ & $\times 10^{-4}$ \\
\hline \multirow{4}{*}{$d_{2}$} & 10 & 20.2 & 21.9 & 21.9 & 0.0 & 24.2 & 24.2 & 0.0 \\
& 15 & 6.6 & 6.6 & 6.6 & 0.0 & 6.8 & 6.8 & 0.0 \\
& 20 & 2.0 & 2.0 & 2.0 & 0.0 & 2.1 & 2.1 & 0.0 \\
\hline \multirow{3}{*}{} & 10 & 51.3 & 55.0 & 54.9 & 0.1 & 57.5 & 57.4 & 0.1 \\
& 15 & 16.9 & 16.9 & 16.8 & 0.0 & 16.8 & 16.8 & 0.0 \\
& 20 & 5.2 & 5.2 & 5.2 & 0.0 & 5.2 & 5.2 & 0.0 \\
\hline
\end{tabular}

TABLE II: Linear FM: CRB, variance and square of the bias of the estimators $d_{k}$ versus SNR $(N=128)$.

is equal to $2.4 \times 10^{-4}$.

Figure 3 presents the estimation errors, the CRBs and the CRB limits (see Remark 2) for the unbalance parameters. We see that the CRBs are correctly bounded by the CRB limits. We also note that the CRBs, the lower and upper CRB limits coincide for particular values of $N(N=42,83,125, \cdots)$. These values correspond to the case where $\mathbf{R}_{\mathbf{x}} \approx 0.5 \mathbf{I}$. Specifically, it can be shown that this equality is satisfied when the number of samples is equal to an integer multiple of the half period $\left(0.5 \times F_{s} / f_{s} \approx 41.66\right)$, or when $N \rightarrow \infty$. Figure 3 also shows that the estimation of $d_{1}$ is more accurate than the estimation of $d_{2}$. Specifically, the CRB of $d_{1}$ is about 2.53 times lower than that of $d_{2}$, and the MSEs follow the same trend for $\mathrm{SNR} \geq 10 \mathrm{~dB}$. This observation is consistent with Remark 3. Indeed, using the expression of $a[n]$ and $\phi[n]$, it can be checked that for large $N$ the covariance matrix tends to $\mathbf{R}_{\mathbf{x}} \approx 0.5 \mathbf{I}$, which implies that $\mathrm{CRB}\left[d_{2}\right] / \operatorname{CRB}\left[d_{1}\right]=\left(\frac{d_{2}}{d_{1}}\right)^{2} \approx 2.5$.

Comparing the MSEs to the CRB in the asymptotic region, we note that the MLEs of $d_{1}$ and $d_{2}$ do not achieve the CRBs at fixed SNR. Indeed, we observe an offset between the MSEs and the CRBs, even with a large number of samples.

Figure 4 shows the MSE, the CRB and the asymptotic CRB for the instantaneous amplitude $a[n]$. For the purpose of comparison, the MSEs obtained with the MLE are compared with those obtained with the Clarke transform and PCA-based techniques [17]. In this figure, we observe that the CRB decreases as the SNR increases. Furthermore, we see that the exact CRB tends to the asymptotic one when $N \rightarrow \infty$. Regarding the estimators, we observe that the Clarke estimator does not provide reliable results due to the unbalance condition. Specifically, the MSE exhibits an "error floor" that is equals to $10^{-2}$ for the 


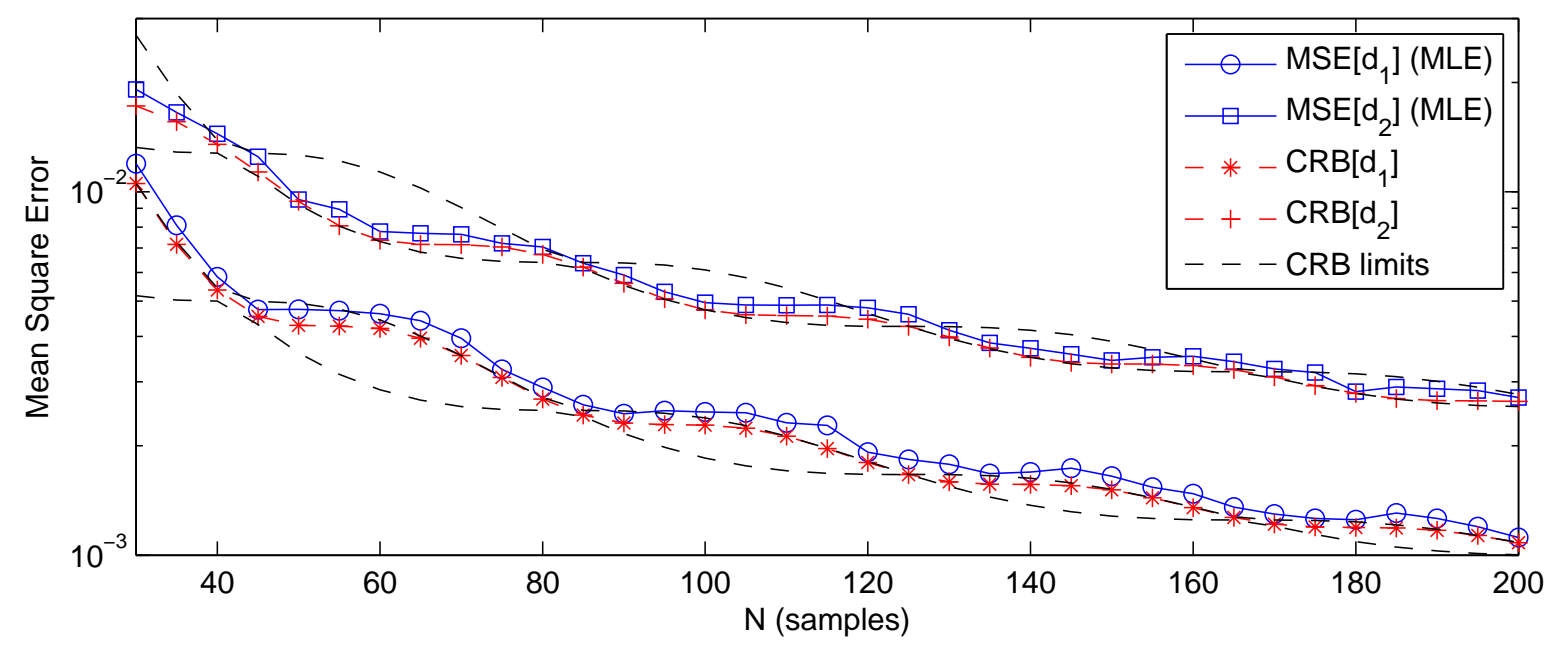

(a) Estimation error versus data length $\left(\sigma^{2}=4.10^{-2}\right)$

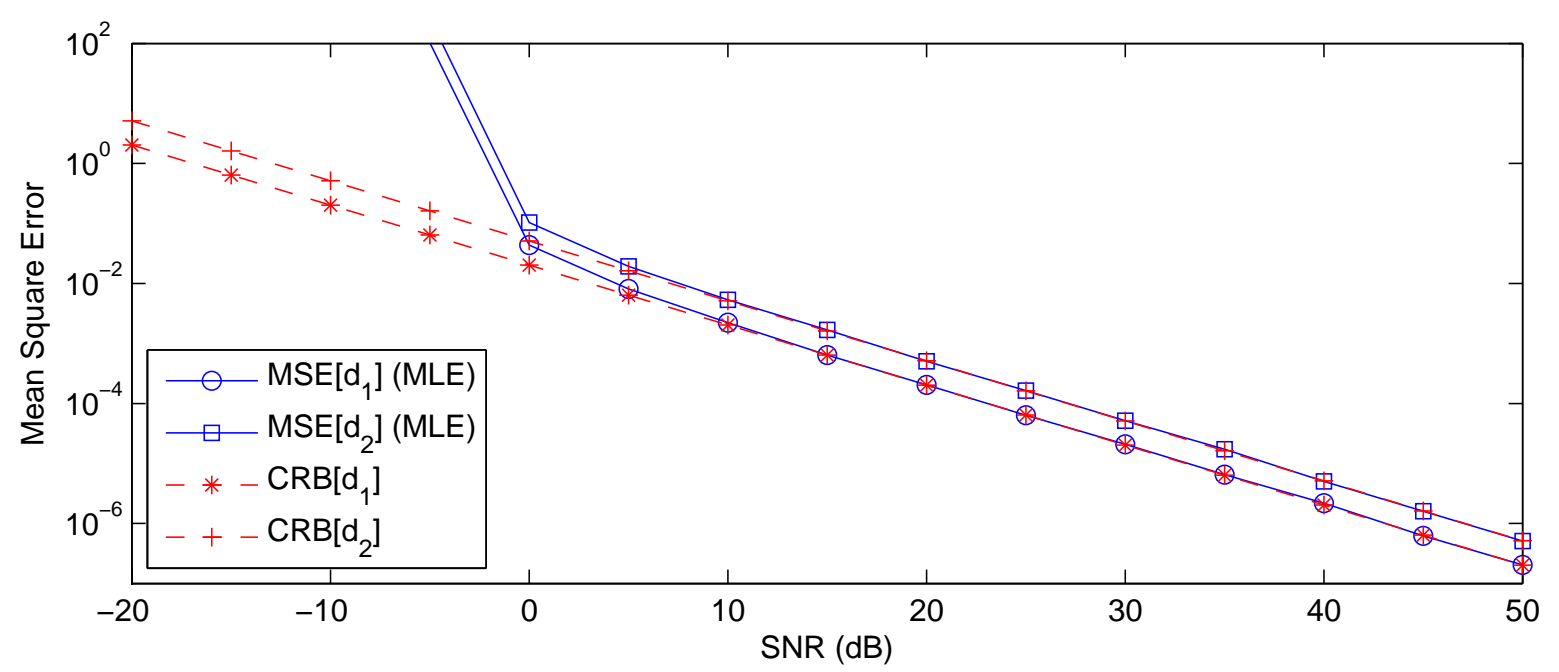

(b) Estimation error versus $\operatorname{SNR}(N=128)$

Fig. 3: Linear FM: Estimation of $d_{1}$ and $d_{2}$

asymptotic case SNR $\rightarrow \infty$. We also note that the performances of the PCA estimator highly depend on the data length. Indeed, this estimator is based on the assumption that $\widehat{\mathbf{R}}_{x}=\sigma_{s}^{2} \mathbf{I}$, which is only satisfied when $N$ is equal to an integer multiple of the half period, or when $N \rightarrow \infty$. Finally, we observe that the MLE outperforms all the other methods and achieves the CRB for $\mathrm{SNR} \rightarrow \infty$.

Figure 5 presents the estimation error and the CRB for the instantaneous phase $\phi[n]$. We can draw similar conclusions as those from Figure 4. Specifically, the MLE outperforms the other estimators and achieves the CRB for SNR $\rightarrow \infty$. Furthermore, we note that the MSE tends to a fixed value at low SNR. 


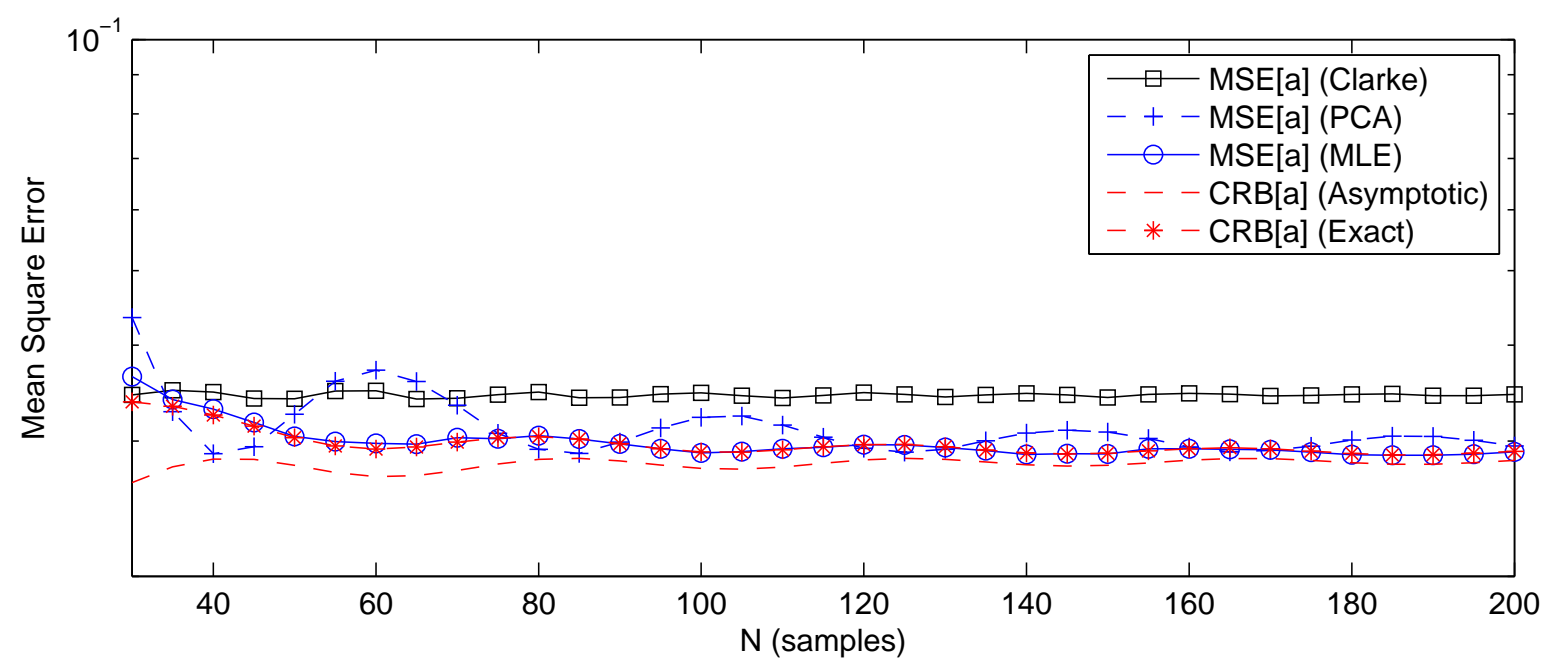

(a) Estimation error versus data length $\left(\sigma^{2}=4.10^{-2}\right)$

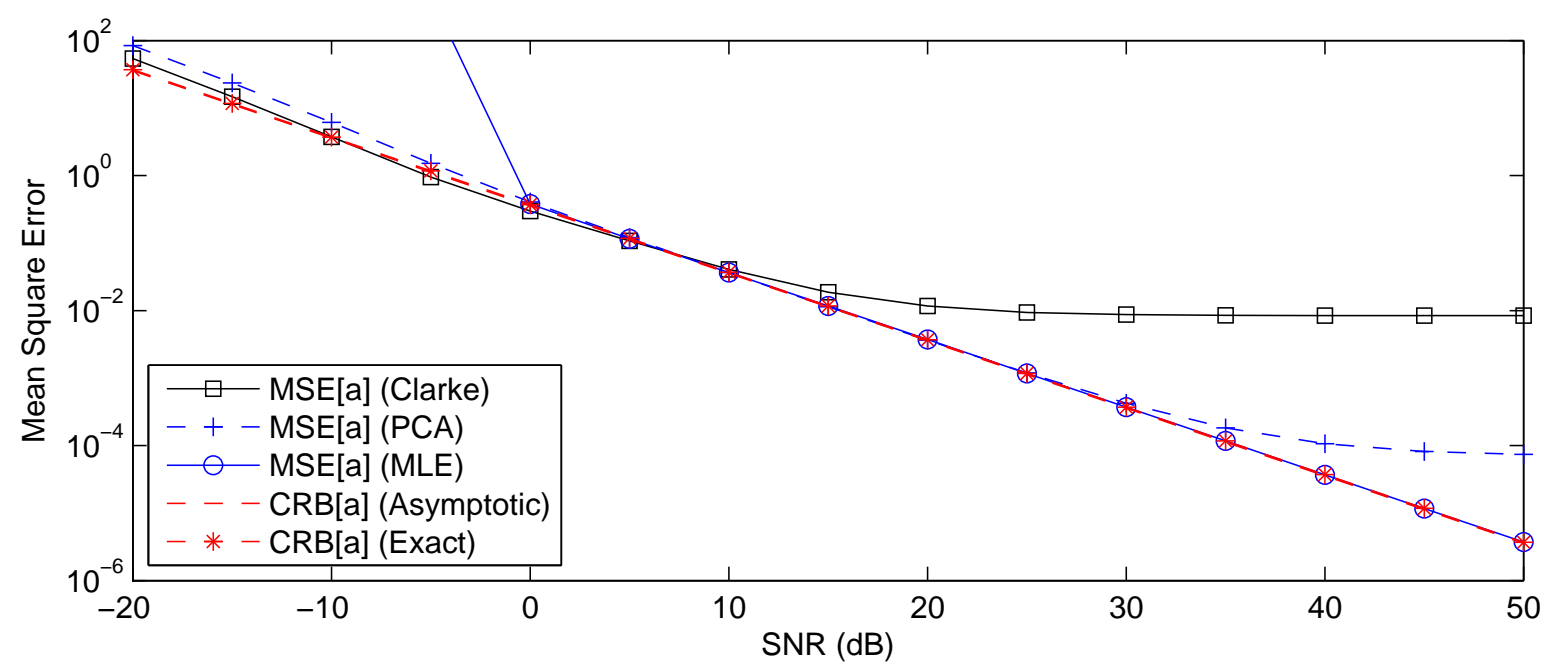

(b) Estimation error versus SNR $(N=128)$

Fig. 4: Linear FM: Estimation of $a[n]$

Specifically, the random variable $[\widehat{\phi}[n]-\phi[n]]_{[-\pi, \pi(}^{2}$ tends to a uniform distribution with support $[-\pi, \pi($ at low $\mathrm{SNR}$, which implies that $\operatorname{MSE}[\phi] \approx \frac{\pi^{2}}{3}$. 


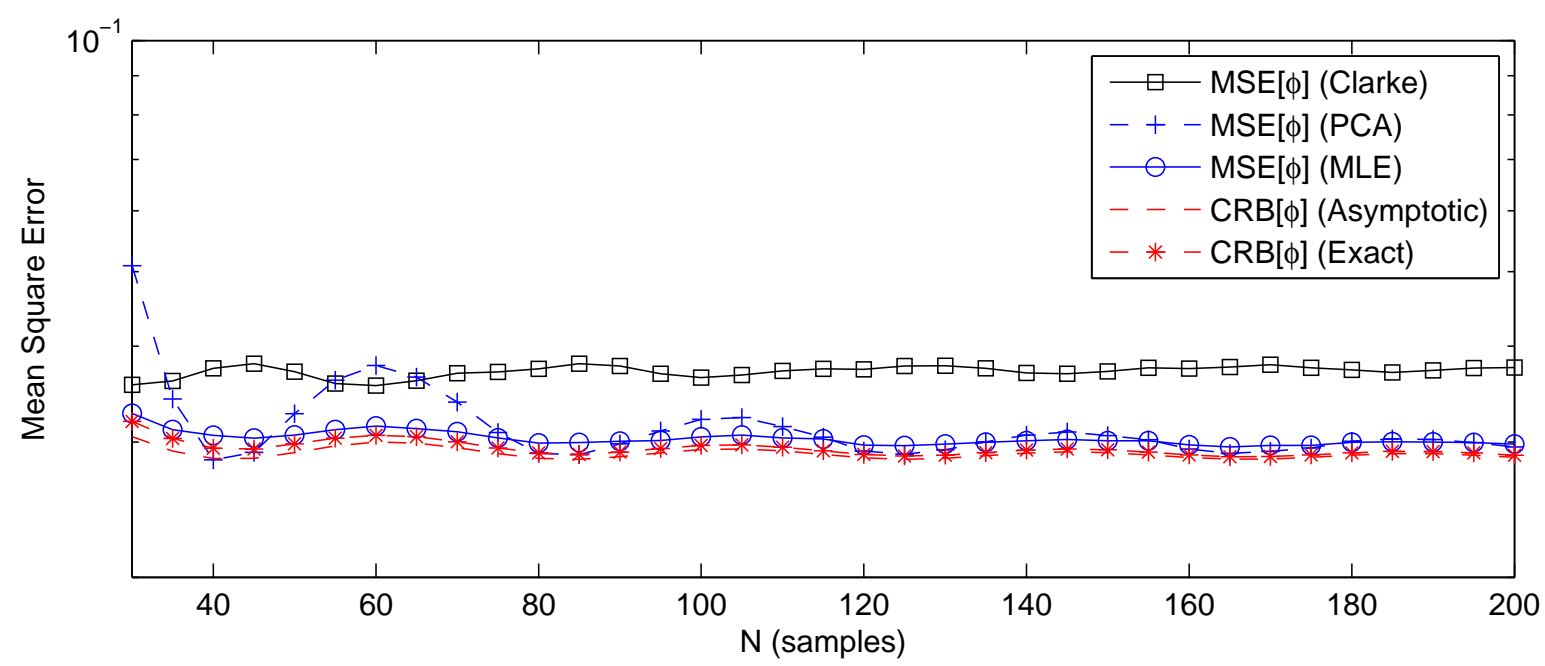

(a) Estimation error versus data length $\left(\sigma^{2}=4.10^{-2}\right)$

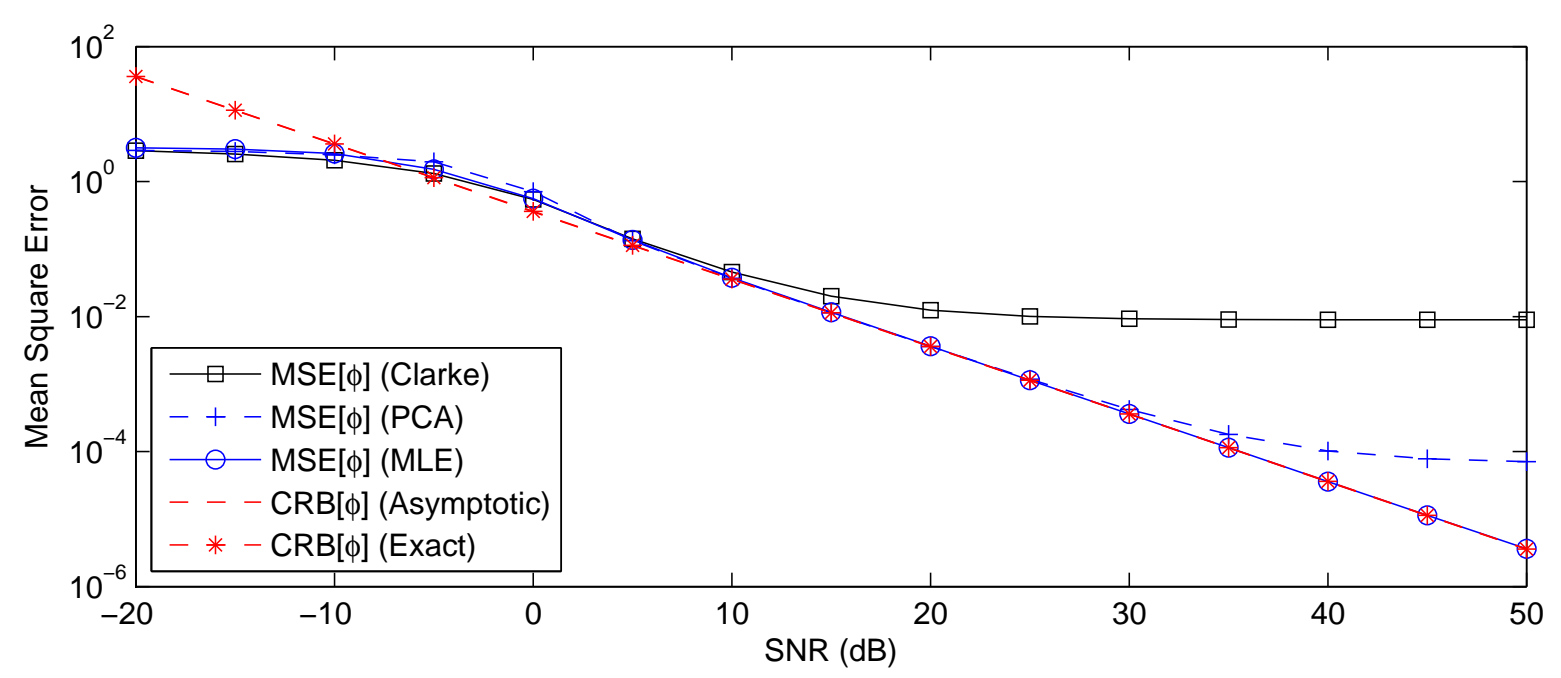

(b) Estimation error versus SNR $(N=128)$

Fig. 5: Linear FM: Estimation of $\phi[n]$

\section{B. Estimation with sinusoidal amplitude and phase modulation}

The performances of the proposed technique are evaluated using three-phase signals with sinusoidal amplitude and phase modulation. Under sinusoidal modulation, IA and IP are given by $[9,5.5 .6]$

$$
\begin{aligned}
& a[n]=1+k_{x} \cos \left(2 \pi f_{m} n / F_{s}\right) \\
& \phi[n]=2 \pi f_{s} n / F_{s}+k_{a} \cos \left(2 \pi f_{m} n / F_{s}+\pi\right)
\end{aligned}
$$


where $k_{x}$ is the amplitude modulation factor and $k_{a}$ is the phase modulation factor. In the following simulations, these parameters are chosen according to the worst scenario of the IEEE Standard C37.118 [9] $\left(k_{x}=k_{a}=0.1\right)$. Figure $2 \mathrm{~b}$ displays the corresponding signals with a modulation frequency of $f_{m}=5 \mathrm{~Hz}$. The evolution of the CRBs and the MSEs versus the data length or the SNR is very similar to the one observed in subsection V-A, and therefore, are not presented here for the sake of brevity. Instead, as recommended in the standard C37.118 [9], the following experiments examine the influence of the modulation frequency $f_{m}$ on the statistical performances.

Figure 6 displays the CRBs and the MSEs of the unbalance parameters for $N=128$ and $\sigma^{2}=4 \times 10^{-2}$. We see that the CRBs and MSEs do not seem to be affected by the modulation frequency. A closer inspection reveals some small non-monotonic variations. For instance, the CRB of $d_{2}$ is equal to 0.0034 at $f_{m}=1 \mathrm{~Hz}$, and to 0.0036 at $f_{m}=10 \mathrm{~Hz}$. These variations are caused by the sample covariance matrix $\mathbf{R}_{\mathbf{x}}$ in (60), which is slightly sensitive to $f_{m}$.

Figure 7 and 8 present the MSEs, the CRBs and the asymptotic CRBs for the IA and IP. As reported before, we observe that these quantities are not significantly affected by $f_{m}$.

\section{Influence of Harmonics}

In this subsection, we analyse the behaviour of the proposed algorithm in the presence of a model mismatch caused by harmonics. In power system, harmonics are produced by nonlinear loads and are a big nuisance in transmission networks [35]. Due to waveform symmetry, electrical signals mostly contain odd harmonics. By adding odd harmonics in (1), we get the following model

$$
\begin{aligned}
y_{k}[n] & =d_{k} a[n] \cos (\phi[n]-2 k \pi / 3) \\
& +\sum_{l=3,5, . .} d_{k} a_{l}[n] \cos (l \phi[n]-2 k l \pi / 3)+b_{k}[n]
\end{aligned}
$$

where $a_{l}[n]$ corresponds to the level of the $l^{t h}$ harmonic. In this subsection, we assume a constant IA, $a[n]=1$, and linear IP, $\phi[n]=2 \pi f_{s} n / F_{s}$. Furthermore, we set $a_{3}[n]=\beta, a_{5}[n]=0.5 \beta$ and $a_{7}[n]=0.3 \beta$. Figures 9, 10 and 11 present the influence of the harmonic coefficient $\beta$ on the MSEs. The CRBs are not presented here due to the model mismatch.

We observe that the MSEs increase as $\beta$ increases. Moreover, it is interesting to note that, while $\operatorname{MSE}\left[d_{2}\right]>\operatorname{MSE}\left[d_{1}\right]$ for small values of $\beta$, this trend is reversed for higher values of $\beta$. Finally, we can note that the PCA estimator of the instantaneous phase, $\phi[n]$, seems to be slightly more robust than the MLE estimator. 


\section{Frequency Estimation with linear FM and sinusoidal AM/PM}

In this experiment, the proposed technique is applied to the estimation of the instantaneous frequency. The instantaneous frequency can be derived from the MLE of the instantaneous phase as follows

$$
\widehat{f}[n]=\frac{F_{s}}{4 \pi}\left(\widehat{\phi}_{u}[n+1]-\widehat{\phi}_{u}[n-1]\right)
$$

where $\widehat{\phi}_{u}[n]$ corresponds to the unwrapped MLE of the instantaneous phase. This frequency estimate is compared to the one obtained with the Augmented Complex Least Mean Square (ACLMS) algorithm in [19] using a perfect initialization, i.e. $f[0]=60 \mathrm{~Hz}$, and a step size of $\mu=0.01$ and $\mu=0.1$. Figure 12 displays the reference and the estimated frequencies with linear frequency modulation (see subsection V-A), and sinusoidal amplitude and phase modulation (see subsection V-B). This figure shows that all the considered estimators are able to track the reference frequency. Concerning the ACLMS, we observe that a large step size $\mu$ is required to obtain a rapid and precise estimate. However, we also note that a large step size results in an overshoot, which can be problematic in monitoring applications. Finally, we see that the MLE gives the best frequency estimate in the two simulations. Nevertheless, this statement must be tempered because the proposed MLE assumes static unbalance parameters (see AS1)), as opposed to the ACLMS. When AS1) is not satisfied, the proposed MLE must be adapted to take into account the non-stationarity of the sample covariance matrix.

\section{CONCLUSION}

In this paper, we investigated the estimation of the amplitude, phase and unbalance parameters in three-phase systems with amplitude unbalance.

First, a new approach to estimate the amplitude, the phase and the unbalance parameters was proposed. We demonstrated that the Maximum Likelihood Estimators of these parameters have simple closed-form expressions. More precisely, the unbalance parameters are computed from the eigenvector associated with the smallest eigenvalue of the sample covariance matrix, and the amplitude and phase are estimated from a linear transformation of the three-phase signal. We also provided a low complexity algorithm to implement these estimators, which is suitable for real-time implementation in embedded systems.

Then, the Cramér-Rao Bounds (CRBs) of the amplitude, phase and unbalance parameters were derived and analysed. We provided explicit expressions for the CRB of the unbalance parameters and asymptotic expressions for the CRB of the amplitude and phase.

The performances of the proposed estimators were validated using synthetic signals compliant with the IEEE Standard C37.118 and compared with other estimators such as the Clarke transform, the PCA 
estimator and the ACLMS. These simulations showed that the proposed estimators outperform the other techniques and achieve the CRB at high SNR, whatever the number of samples and the modulation frequency. Extension of the proposed technique to more complicated signals, including non-sinusoidal signals with amplitude and phase unbalance, will be investigated in future works.

\section{REFERENCES}

[1] Y.-F. Huang, S. Werner, J. Huang, N. Kashyap, and V. Gupta, "State estimation in electric power grids: Meeting new challenges presented by the requirements of the future grid," IEEE Signal Processing Magazine, vol. 29, no. 5, pp. 33-43, 2012.

[2] A. Kwasinski and A. Kwasinski, "Signal processing in the electrification of vehicular transportation," IEEE Signal Processing Magazine, vol. 29, no. 5, pp. 14-23, 2012.

[3] P. Top, M. Bell, E. Coyle, and O. Wasynczuk, "Observing the power grid: Working toward a more intelligent, efficient, and reliable smart grid with increasing user visibility," IEEE Signal Processing Magazine, vol. 29, no. 5, pp. 24-32, 2012.

[4] M. Benbouzid, "A review of induction motors signature analysis as a medium for faults detection," IEEE Transactions on Industrial Electronics, vol. 47, no. 5, pp. 984-993, 2000.

[5] M. Blodt, M. Chabert, J. Regnier, and J. Faucher, "Mechanical load fault detection in induction motors by stator current time-frequency analysis," IEEE Transactions on Industry Applications, vol. 42, no. 6, pp. 1454-1463, 2006.

[6] M. Blodt, D. Bonacci, J. Regnier, M. Chabert, and J. Faucher, "On-line monitoring of mechanical faults in variable-speed induction motor drives using the Wigner distribution," IEEE Transactions on Industrial Electronics, vol. 55, no. 2, pp. 522-533, 2008.

[7] M. Blodt, J. Regnier, and J. Faucher, "Distinguishing load torque oscillations and eccentricity faults in induction motors using stator current Wigner distributions," IEEE Transactions on Industry Applications, vol. 45, no. 6, pp. 1991-2000, 2009.

[8] A. Bouzida, O. Touhami, R. Ibtiouen, A. Belouchrani, M. Fadel, and A. Rezzoug, "Fault diagnosis in industrial induction machines through discrete wavelet transform," IEEE Transactions on Industrial Electronics, vol. 58, no. 9, pp. 4385-4395, 2011.

[9] "IEEE Standard for Synchrophasor Measurements for Power Systems," IEEE Std C37.118.1-2011 (Revision of IEEE Std C37.118-2005), pp. 1-61, 2011.

[10] D. Vakman, "On the Analytic Signal, the Teager-Kaiser Energy Algorithm, and Other Methods for Defining Amplitude and Frequency," IEEE Transactions on Signal Processing, vol. 44, no. 4, pp. 791-797, 1996.

[11] L. Marple Jr, "Computing the discrete-time analytic signal via FFT," IEEE Transactions on Signal Processing, vol. 47, no. 9, pp. 2600-2603, 1999.

[12] E. Bedrosian, "A product theorem for Hilbert transforms," Proceedings of the IEEE, vol. 51, pp. 868-869, 1963.

[13] B. Picinbono, “On instantaneous amplitude and phase of signals," IEEE Transactions on Signal Processing, vol. 45, no. 3, pp. 552-560, 1997.

[14] E. Clarke, Circuit analysis of AC power systems; symmetrical and related components. Wiley, 1943, vol. 1.

[15] A. K. Pradhan, A. Routray, and A. Basak, "Power System Frequency Estimation Using Least Mean Square Technique," IEEE Transactions on Power Delivery, vol. 20, no. 3, pp. 1812-1816, 2005. 
[16] M. M. Canteli, A. O. Fernandez, L. I. Eguiluz, and C. R. Estebanez, “Three-phase adaptive frequency measurement based on Clarke's transformation," IEEE Transaction on Power Delivery, vol. 21, no. 2, 2006.

[17] V. Choqueuse, M. Benbouzid, Y. Amirat, and S. Turri, "Diagnosis of three-phase electrical machines using multidimensional demodulation techniques," IEEE Transactions on Industrial Electronics, vol. 59, no. 4, pp. 2014 -2023, 2012.

[18] Y. Xia and D. Mandic, "Widely Linear Adaptive Frequency Estimation of Unbalanced Three-Phase Power Systems," IEEE Transactions on Instrumentation and Measurement, vol. 61, no. 1, pp. 74-83, 2012.

[19] Y. Xia, S. Douglas, and D. Mandic, “Adaptive frequency estimation in smart grid applications," IEEE Signal Processing Magazine, vol. 5, pp. 44-54, 2012.

[20] A. V. Jouanne, “Assessment of voltage unbalance,” IEEE Transactions on Power Delivery, vol. 16, no. 4, pp. 782-790, 2001.

[21] "IEEE Standard Definitions for the Measurement of Electric Power Quantities Under Sinusoidal, Nonsinusoidal, Balanced, or Unbalanced Conditions," IEEE Std 1459-2010 (Revision of IEEE Std 1459-2000), pp. 1-40, 2010.

[22] T. Tayjasanant, C. Li, and W. Xu, "A resistance sign-based method for voltage sag source detection," IEEE Transactions on Power Delivery, vol. 20, no. 4, pp. 2544-2551, 2005.

[23] P. Stoica and P. Babu, "The Gaussian Data Assumption Leads to the Largest Cramér-Rao Bound," IEEE Signal Processing Magazine, no. May, pp. 132-133, 2011.

[24] P. Stoica and R. Moses, Introduction to Spectral Analysis. Prentice-Hall, 1997.

[25] P. Stoica and A. Nehorai, "Performance study of conditional and unconditional direction-of-arrival estimation," IEEE Transactions on Acoustics, Speech and Signal Processing, vol. 38, no. 10, pp. 1783-1795, 1990.

[26] H. Krim and M. Viberg, "Two decades of array signal processing research, the parametric approach," IEEE Signal Processing Magazine, pp. 67-94, 1996.

[27] R. Horn and C. Johnson, Matrix Analysis. Cambridge University Press, 1990.

[28] S. Kay, Fundamentals of statistical signal processing: estimation theory. Prentice-Hall, 1993.

[29] J. Kopp, "Efficient numerical diagonalization of hermitian $3 \times 3$ matrices," International Journal of Modern Physics C, vol. 19, no. 03, pp. 523-548, 2008.

[30] B. Ottersten, M. Viberg, P. Stoica, and A. Nehorai, Exact and large sample maximum likelihood techniques for parameter estimation and detection in array processing. Springer, 1993.

[31] A. Renaux, P. Forster, E. Chaumette, and P. Larzabal, "On the high-snr conditional maximum-likelihood estimator full statistical characterization," IEEE Transactions on Signal Processing, vol. 54, no. 12, pp. 4840-4843, 2006.

[32] A. Renaux, P. Forster, E. Boyer, and P. Larzabal, "Unconditional maximum likelihood performance at finite number of samples and high signal-to-noise ratio,” IEEE Transactions on Signal Processing, vol. 55, no. 5, pp. 2358-2364, 2007.

[33] P. Stoica and A. Nehorai, "MUSIC, maximum likelihood, and cramer-rao bound," IEEE Transactions on Acoustics. Speech. and Signal Processing, vol. 37, no. 5, pp. 720-741, 1989.

[34] Y. Xia and D. Mandic, "Augmented mvdr spectrum-based frequency estimation for unbalanced power systems," IEEE Transactions on Instrumentation and Measurement, vol. 62, no. 7, pp. 1917-1926, 2013.

[35] M. Begovic, P. Djuric, S. Dunlap, and A. Phadke, "Frequency tracking in power networks in the presence of harmonics," IEEE Transactions on Power Delivery, vol. 8, no. 2, pp. 480-486, 1993.

[36] G. Marsaglia, "Ratios of normal variables," Journal of Statistical Software, vol. 16, no. 4, pp. 1-10, 2006.

[37] G. Van Kempen and L. Van Vliet, "Mean and variance of ratio estimators used in fluorescence ratio imaging," Cytometry, vol. 39, no. 4, pp. 300-305, 2000. 


\section{APPENDIX A \\ ASYMPTOTIC PROPERTIES OF $\widehat{d}_{k}$}

Under the assumptions AS1)-AS3), the covariance matrix, $\mathbf{R}$, is given by

$$
\mathbf{R} \triangleq E\left[\mathbf{y}[n] \mathbf{y}^{T}[n]\right]=\mathbf{D H R}_{x} \mathbf{H}^{T} \mathbf{D}^{T}+\sigma^{2} \mathbf{I}
$$

This matrix can be decomposed as

$$
\mathbf{R}=\mathbf{S} \Lambda \mathbf{S}^{T}
$$

where $\Lambda=\operatorname{diag}\left(\lambda_{0}, \lambda_{1}, \sigma^{2}\right)\left(\lambda_{0} \geq \lambda_{1} \geq \sigma^{2}\right)$ is a $3 \times 3$ diagonal matrix containing the eigenvalues and $\mathbf{S}=\left[\mathbf{s}_{0}, \mathbf{s}_{1}, \mathbf{u}\right]$ is a $3 \times 3$ matrix containing the unit-norm associated eigenvectors.

Similarly, the sample covariance matrix, $\widehat{\mathbf{R}}$, can be decomposed as

$$
\widehat{\mathbf{R}}=\widehat{\mathbf{S}} \widehat{\Lambda} \widehat{\mathbf{S}}^{T}
$$

where $\widehat{\mathbf{S}}=\left[\widehat{\mathbf{s}}_{0}, \widehat{\mathbf{s}}_{1}, \widehat{\mathbf{u}}\right]$ and $\Lambda=\operatorname{diag}\left(\widehat{\lambda}_{0}, \widehat{\lambda}_{1}, \widehat{\lambda}_{2}\right)$.

Let us focus on the unit-norm eigenvector, $\widehat{\mathbf{u}}$. For large $N$, $\widehat{\mathbf{u}}$ is approximately jointly Gaussian distributed with mean and covariance matrix given by (see [33])

$$
\begin{gathered}
E[\widehat{\mathbf{u}}] \approx \mathbf{u} \\
\boldsymbol{\Sigma}=E\left[(\widehat{\mathbf{u}}-\mathbf{u})(\widehat{\mathbf{u}}-\mathbf{u})^{T}\right] \approx \frac{\sigma^{2}}{N} \mathbf{C}
\end{gathered}
$$

where

$$
\mathbf{C}=\sum_{k=0}^{1} \frac{\lambda_{k}}{\left(\lambda_{k}-\sigma^{2}\right)^{2}} \mathbf{s}_{k} \mathbf{s}_{k}^{T} .
$$

Similarly to (23), let us decompose $\mathbf{u}$ as

$$
\mathbf{u} \triangleq\left[\begin{array}{l}
u_{0} \\
u_{1} \\
u_{2}
\end{array}\right] .
$$

Using this decomposition and (73), the eigenvector $\widehat{u}_{k}$ is approximately distributed as

$$
\widehat{u}_{k} \sim \mathcal{N}\left(u_{k}, \boldsymbol{\Sigma}_{u u}\right)
$$

where $\boldsymbol{\Sigma}_{u v}$ corresponds to the $[u, v]$ element of $\boldsymbol{\Sigma}$. As $\widehat{d}_{k}=\widehat{u}_{0} / \widehat{u}_{k}$, it follows that $\widehat{d}_{k}$ is a ratio of two Gaussian distributions. In the general case, the distribution of a ratio of two Gaussian distributions is quite complicated [36]. However, under the assumption that $\widehat{u}_{k}>0$, which is motivated by (24), it has been shown in [36] that this distribution can be well approximated by a Gaussian distribution. Furthermore, 
using Taylor expansions, the mean and variance of a ratio of two random variables can be approximated by (see for example [37])

$$
\begin{aligned}
E\left[\frac{\widehat{u}_{0}}{\widehat{u}_{k}}\right] & \approx \frac{E\left[\widehat{u}_{0}\right]}{E\left[\widehat{u}_{k}\right]}-\frac{\boldsymbol{\Sigma}_{0 k}}{E^{2}\left[\widehat{u}_{k}\right]}+\frac{E\left[\widehat{u}_{0}\right] \boldsymbol{\Sigma}_{k k}}{E^{3}\left[\widehat{u}_{k}\right]} \\
\operatorname{var}\left(\frac{\widehat{u}_{0}}{\widehat{u}_{k}}\right) & \approx \frac{\boldsymbol{\Sigma}_{00}}{E^{2}\left[\widehat{u}_{k}\right]}-2 \frac{E\left[\widehat{u}_{0}\right] \boldsymbol{\Sigma}_{0 k}}{E^{3}\left[\widehat{u}_{k}\right]}+\frac{E^{2}\left[\widehat{u}_{0}\right] \boldsymbol{\Sigma}_{k k}}{E^{4}\left[\widehat{u}_{k}\right]}
\end{aligned}
$$

Using (25) and (73) in (76), it follows that the bias and variance can be approximated by

$$
\begin{aligned}
& \operatorname{bias}\left(d_{k}\right) \approx \frac{\sigma^{2}}{N u_{k}^{2}}\left(d_{k} \mathbf{C}_{k k}-\mathbf{C}_{0 k}\right) \\
& \operatorname{var}\left(\widehat{d}_{k}\right) \approx \frac{\sigma^{2}}{N u_{k}^{2}}\left(\mathbf{C}_{00}-2 d_{k} \mathbf{C}_{0 k}+d_{k}^{2} \mathbf{C}_{k k}\right)
\end{aligned}
$$

Finally, as $\mathbf{u}=\mathbf{v}(\mathbf{d}) /\|\mathbf{v}(\mathbf{d})\|$, we get (26).

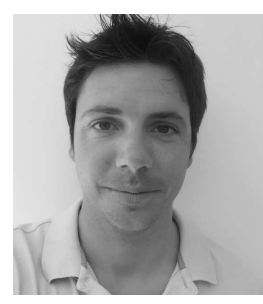

Vincent Choqueuse (M'08) was born in Brest, France, in 1981. He received the Dipl.-Ing. and the M.Sc. degrees in 2004 and 2005, respectively, from the Troyes University of Technology (UTT), Troyes, France, and the Ph.D. degree in 2008 from the University of Brest, Brest, France. Since September 2009, he has been an Associate Professor with the Institut Universitaire de Technologie of Brest, University of Brest, and a member of the Mechanics and Systems Laboratory of Brest (LBMS), EA 4325, France. His research interests include signal processing and statistics for power systems monitoring, Smart-Grid, digital communication and digital audio. Dr. Choqueuse has also developed a free web application for online signal processing and analysis: www.sp4mass.com. 
This is the author's version of an article that has been published in this journal. Changes were made to this version by the publisher prior to publication.

The final version of record is available at http://dx.doi.org/10.1109/TSP.2014.2333565

IEEE TRANSACTIONS ON SIGNAL PROCESSING

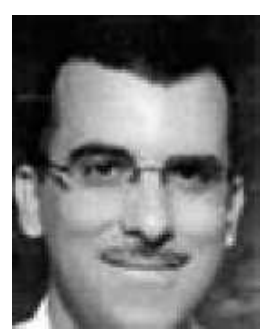

Adel Belouchrani was born in Algiers, Algeria, on May 5, 1967. He received the State Engineering degree in 1991 from Ecole Nationale Polytechnique (ENP), Algiers, the M.S. degree in signal processing from the Institut National Polytechnique de Grenoble(INPG), France, in 1992, and the Ph.D. degree in signal and image processing from Tlcom Paris (ENST), France, in 1995. He was a Visiting Scholar at the Electrical Engineering and Computer Sciences Department, University of California, Berkeley, from 1995 to 1996. He was with the Department of Electrical and Computer Engineering, Villanova University, Villanova, PA, as a Research Associate from 1996 to 1997. From 1998 to 2005, he has been with the Electrical Engineering Department of ENP as Associate Professor.

Since 2006, he has been a Full Professor at ENP. His research interests are in statistical signal processing, (blind) array signal processing, time-frequency analysis and time-frequency array signal processing with applications in biomedical and communications.

Dr. Belouchrani is currently an Associate Editor of the IEEE Transactions on Signal Processing. For more details see his homepage at perso.enp.edu.dz/belouchrani

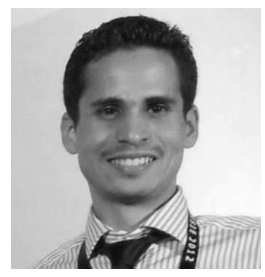

El Houssin El Bouchikhi was born in Khemisset, Morocco, in 1987. He received the Dipl.-Ing. and the M.Sc. Degrees in automatic and electrical engineering from the National Polytechnic Institute of Toulouse (INP-ENSEEIHT), Toulouse, France, in 2010, and the Ph.D. degree in 2012 from the University of Brest, Brest, France. Since September 2012, he has been a post-doctoral scholar with the Institut Superieur de l'Electronique et du Numerique, Brest, France, and a member of the Mechanics and Systems Laboratory of Brest (LBMS), EA 4325, France.

His current research interests include electrical machines fault detection and diagnosis through electrical quantities, especially under nontationary operating conditions 
This is the author's version of an article that has been published in this journal. Changes were made to this version by the publisher prior to publication.

The final version of record is available at http://dx.doi.org/10.1109/TSP.2014.2333565

IEEE TRANSACTIONS ON SIGNAL PROCESSING

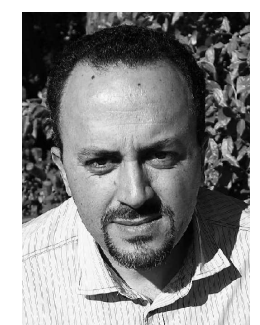

Mohamed El Hachemi Benbouzid (S'92-M'95-SM'98) was born in Batna, Algeria, in 1968. He received the B.Sc. degree in electrical engineering from the University of Batna, Batna, Algeria, in 1990, the M.Sc. and Ph.D. degrees from the National Polytechnic Institute of Grenoble, Grenoble, France, in 1991 and 1994, respectively, both in electrical and computer engineering, and the Habilitation 'a Diriger des Recherches degree from the University of Picardie 'Jules Verne,' Amiens, France, in 2000. After receiving the Ph.D. degree, he joined the Professional Institute of Amiens, University of Picardie 'Jules Verne,' where he was an Associate Professor of Electrical and Computer Engineering. Since September 2004, he has been with the Institut Universitaire de Technologie of Brest, University of Brest, Brest, France, where he is a Professor of Electrical Engineering. His main research interests and experience include analysis, design, and control of electric machines, variable-speed drives for traction, propulsion, and renewable energy applications, and fault diagnosis of electric machines.

Dr. Benbouzid is a Senior Member of the Industrial Electronics, Industry Applications, Power Electronics, and Vehicular Technology Societies. He is an Associate Editor of the IEEE TRANSACTIONS ON ENERGy CONVERSION, the IEEE TRANSACTIONS on Industrial Electronics, the IEEE Transactions on Sustainable EnERGy, and the IEEE Transactions on Vehicular Technology. He was an Associate Editor of the IEEE/ASME Transactions on Mechatronics, from 2006 to 2009 . 


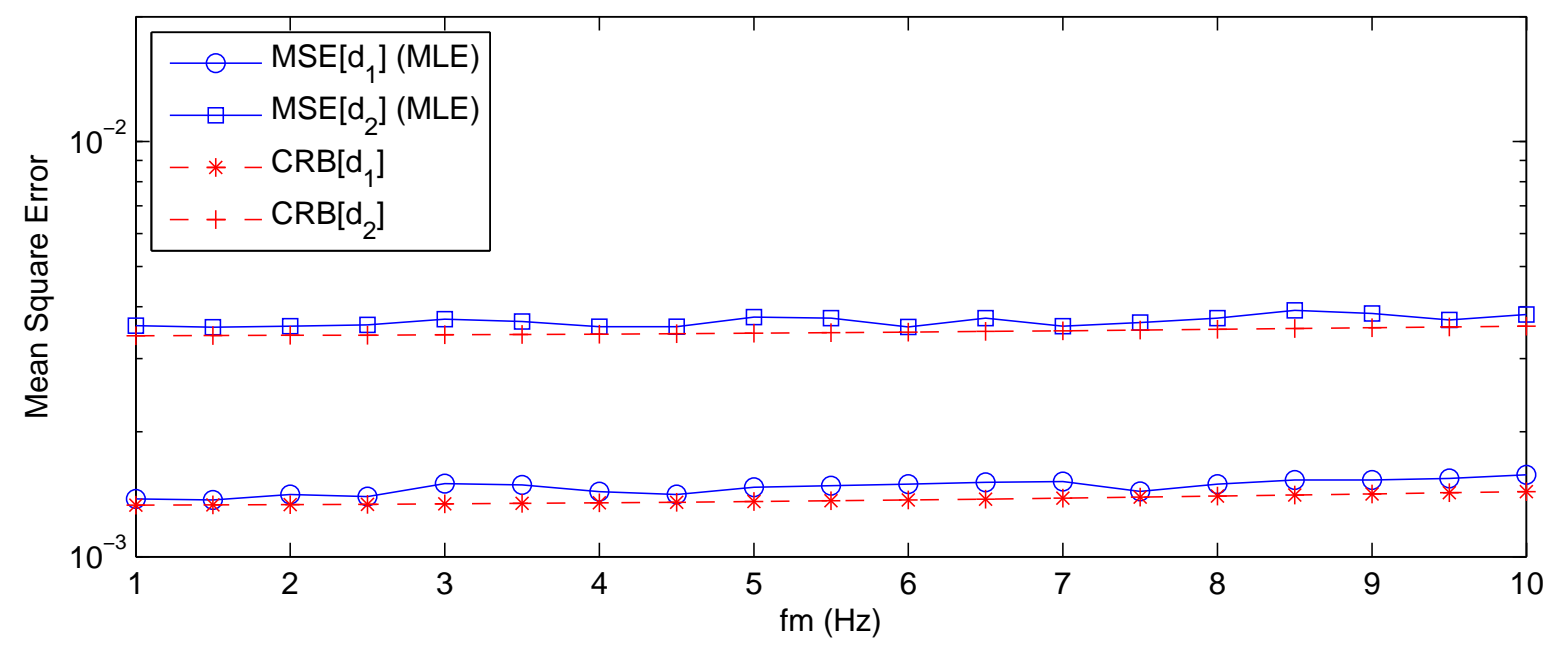

Fig. 6: Sinusoidal AM-PM: Estimation of $d_{1}$ and $d_{2}\left(k_{x}=k_{a}=0.1, N=128\right.$ and $\left.\sigma^{2}=4 \times 10^{-2}\right)$

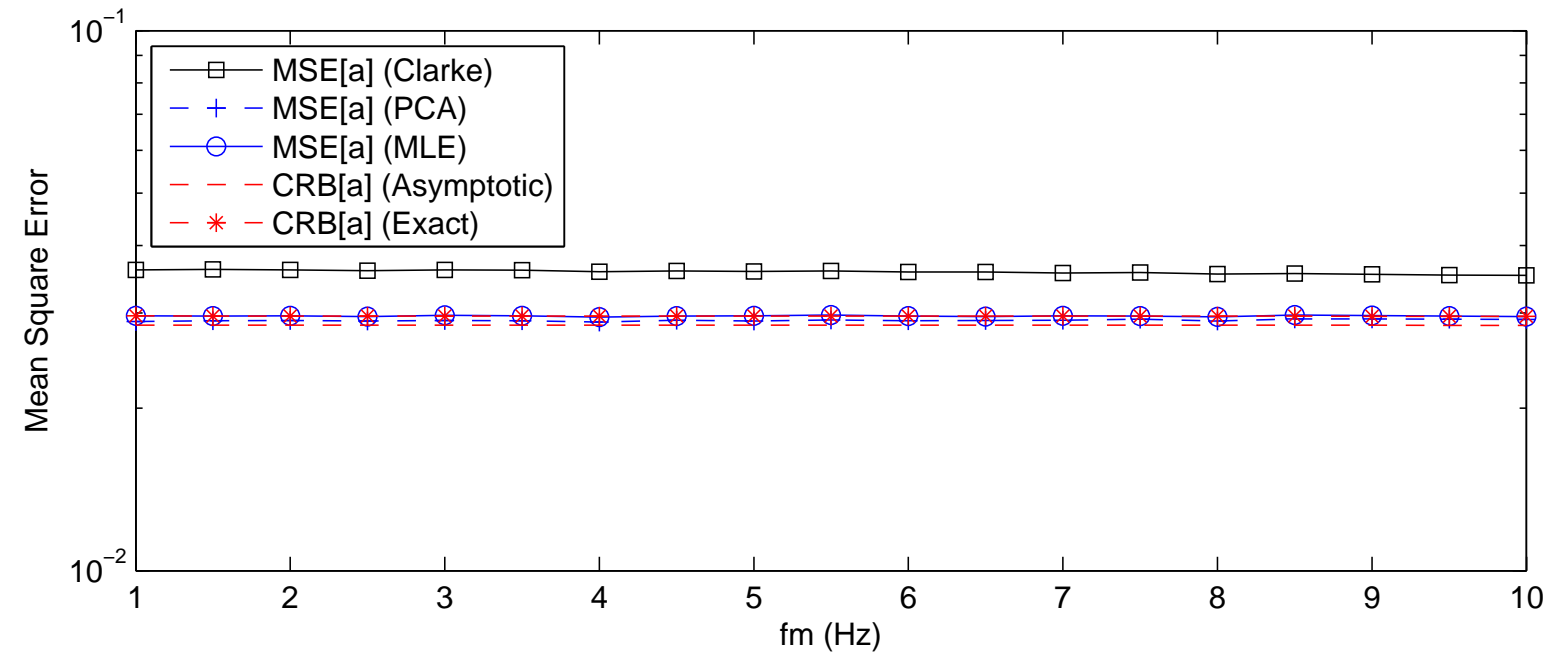

Fig. 7: Sinusoidal AM-PM: Estimation of $a[n]\left(k_{x}=k_{a}=0.1, N=128\right.$ and $\left.\sigma^{2}=4 \times 10^{-2}\right)$

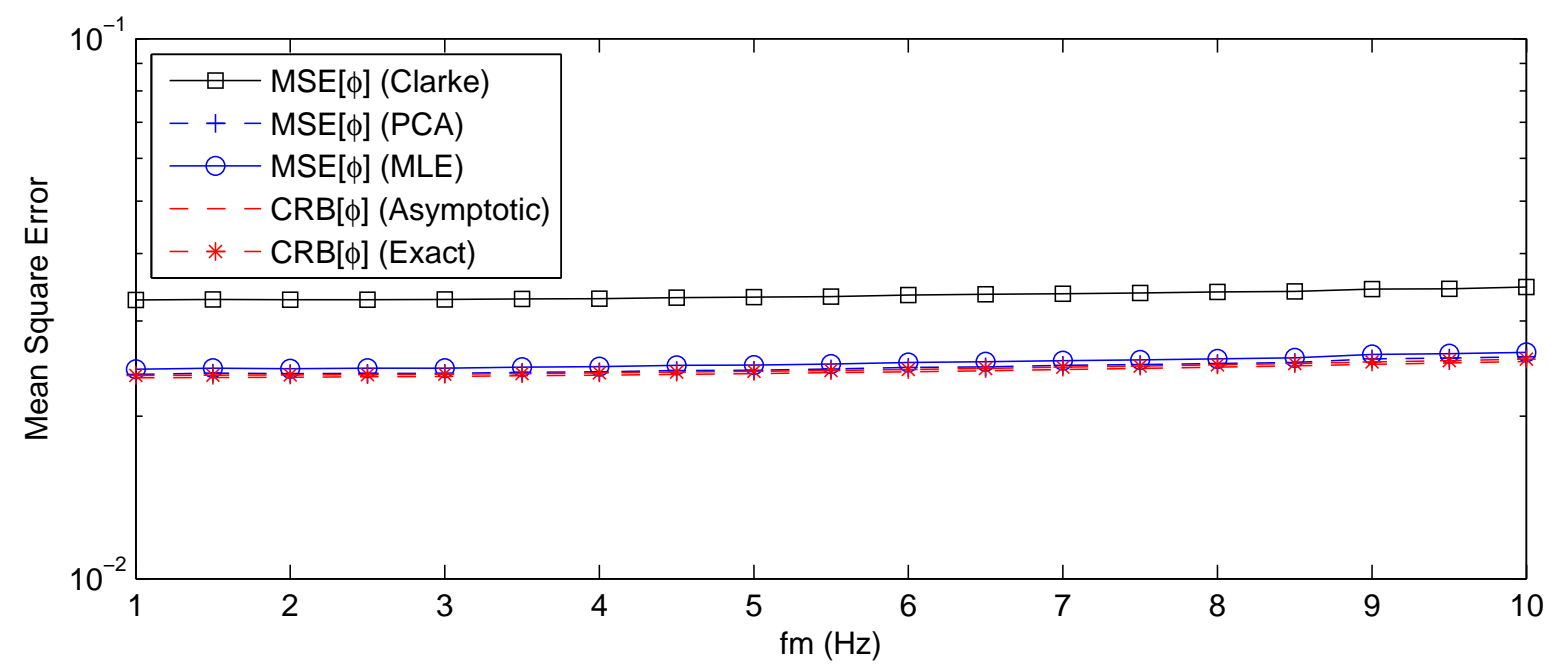

Fig. 8: Sinusoidal AM-PM: Estimation of $\phi[n]\left(k_{x}=k_{a}=0.1, N=128\right.$ and $\left.\sigma^{2}=4 \times 10^{-2}\right)$ June 23, 2014 


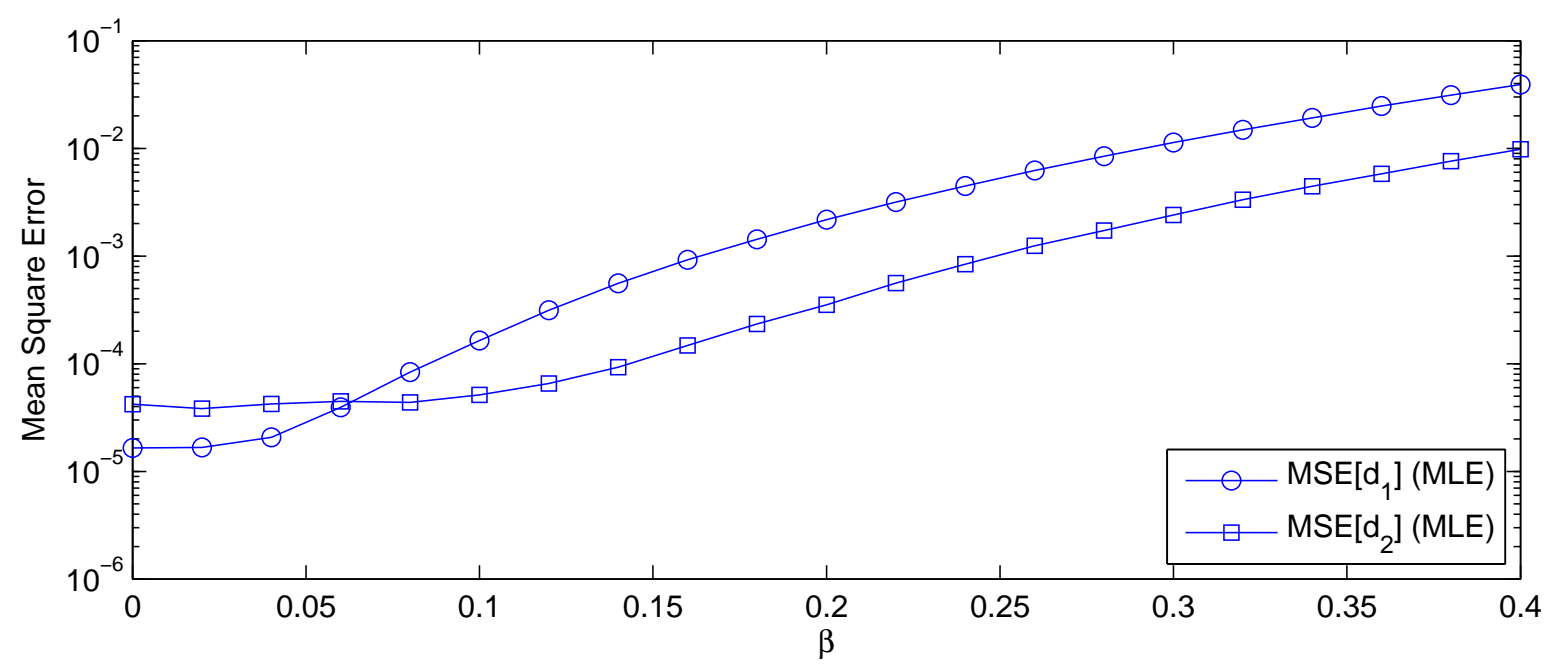

Fig. 9: Influence of harmonics: Estimation of $d_{1}$ and $d_{2}\left(N=128\right.$ and $\left.\sigma^{2}=4 \times 10^{-4}\right)$

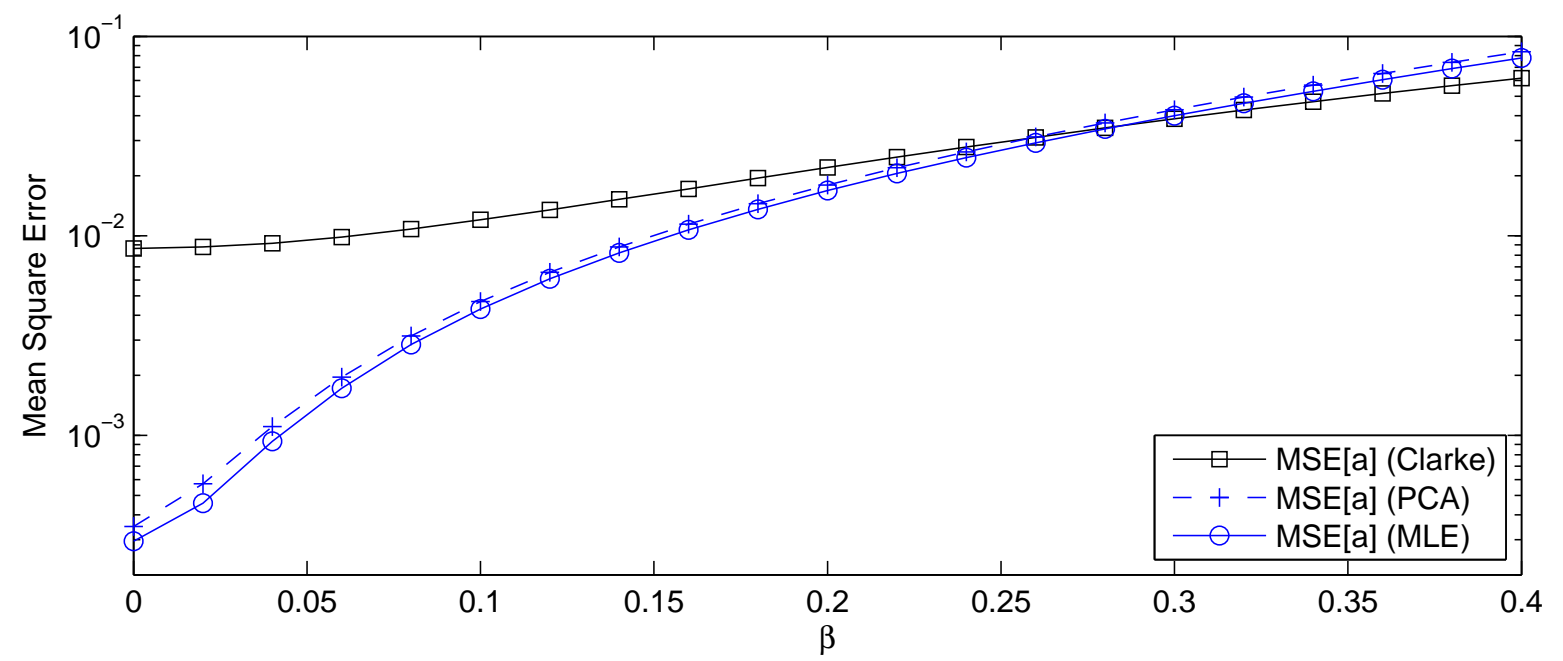

Fig. 10: Influence of harmonics: Estimation of $a[n]\left(N=128\right.$ and $\left.\sigma^{2}=4 \times 10^{-4}\right)$

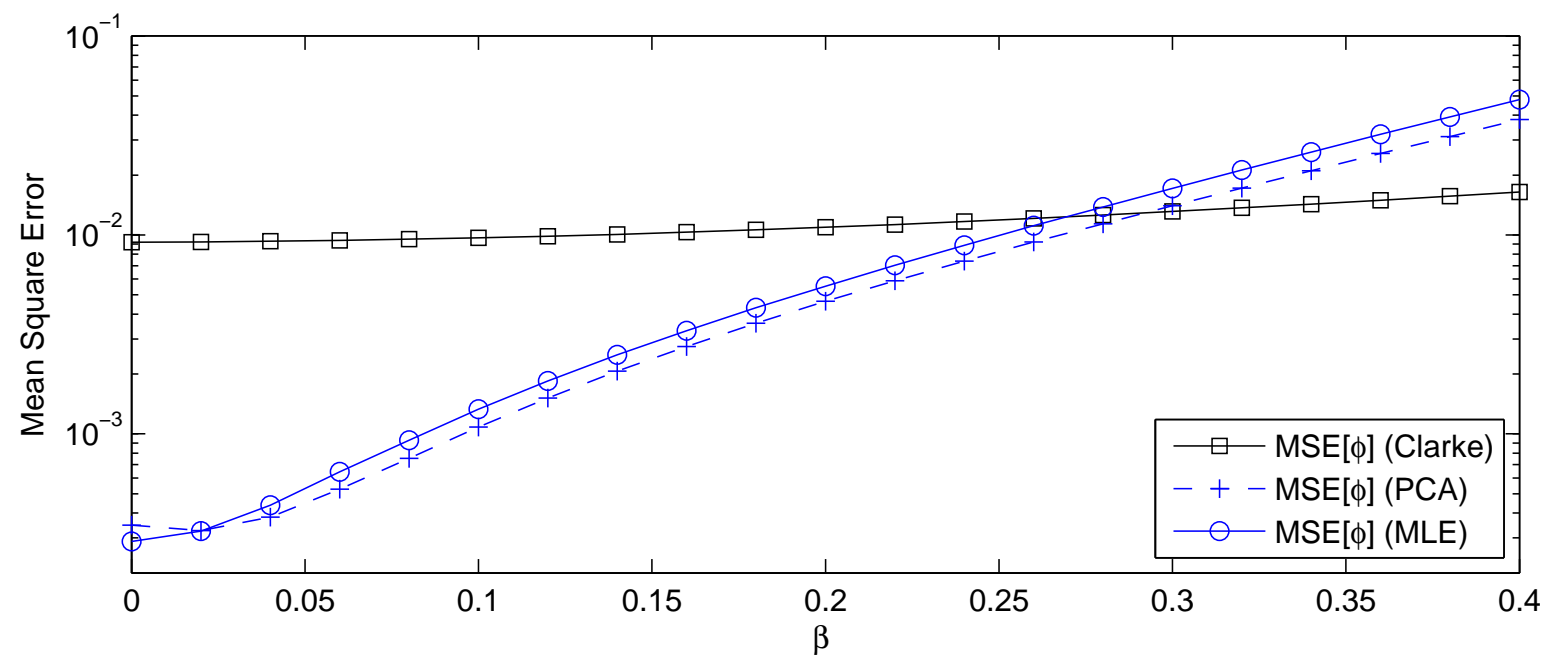

Fig. 11: Influence of harmonics: Estimation of $\phi[n]\left(N=128\right.$ and $\left.\sigma^{2}=4 \times 10^{-4}\right)$ 


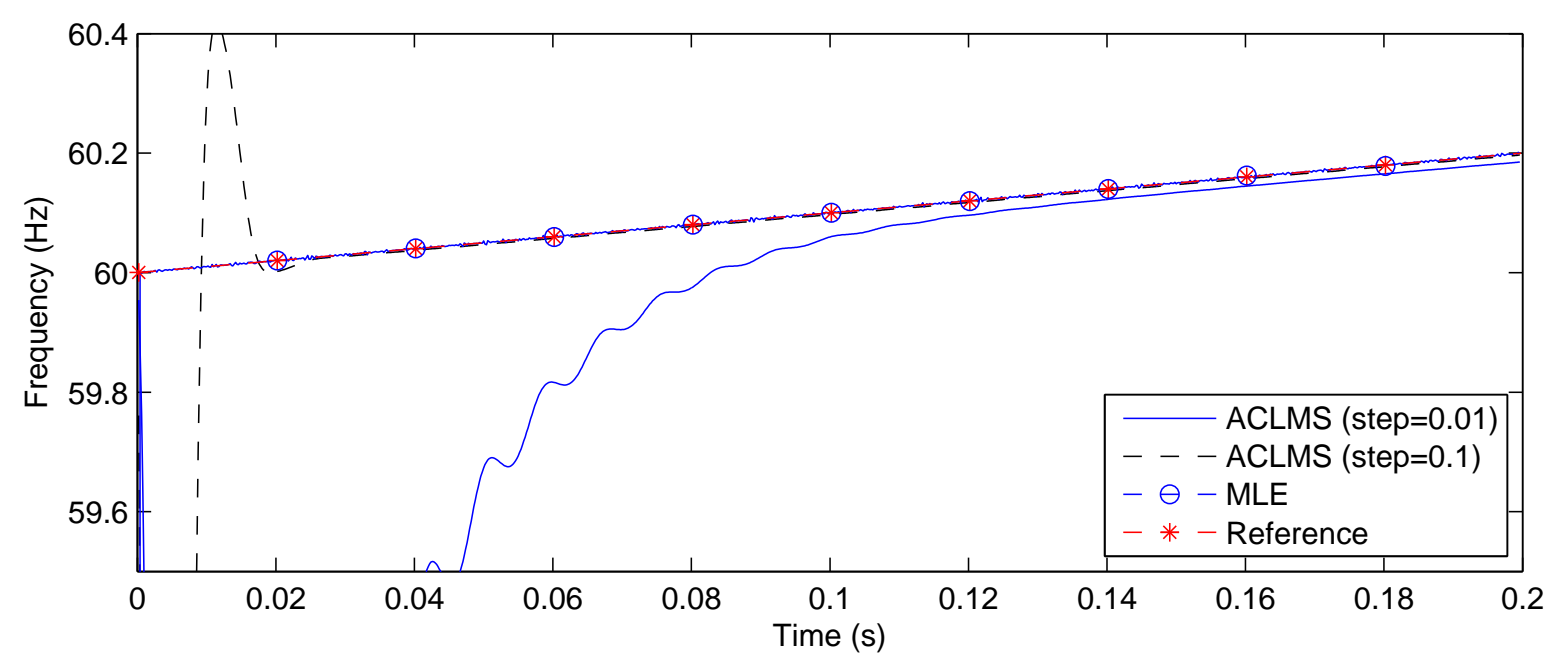

(a) Linear frequency modulation $\left(r_{f}=1 \mathrm{~Hz} / \mathrm{s}\right)$

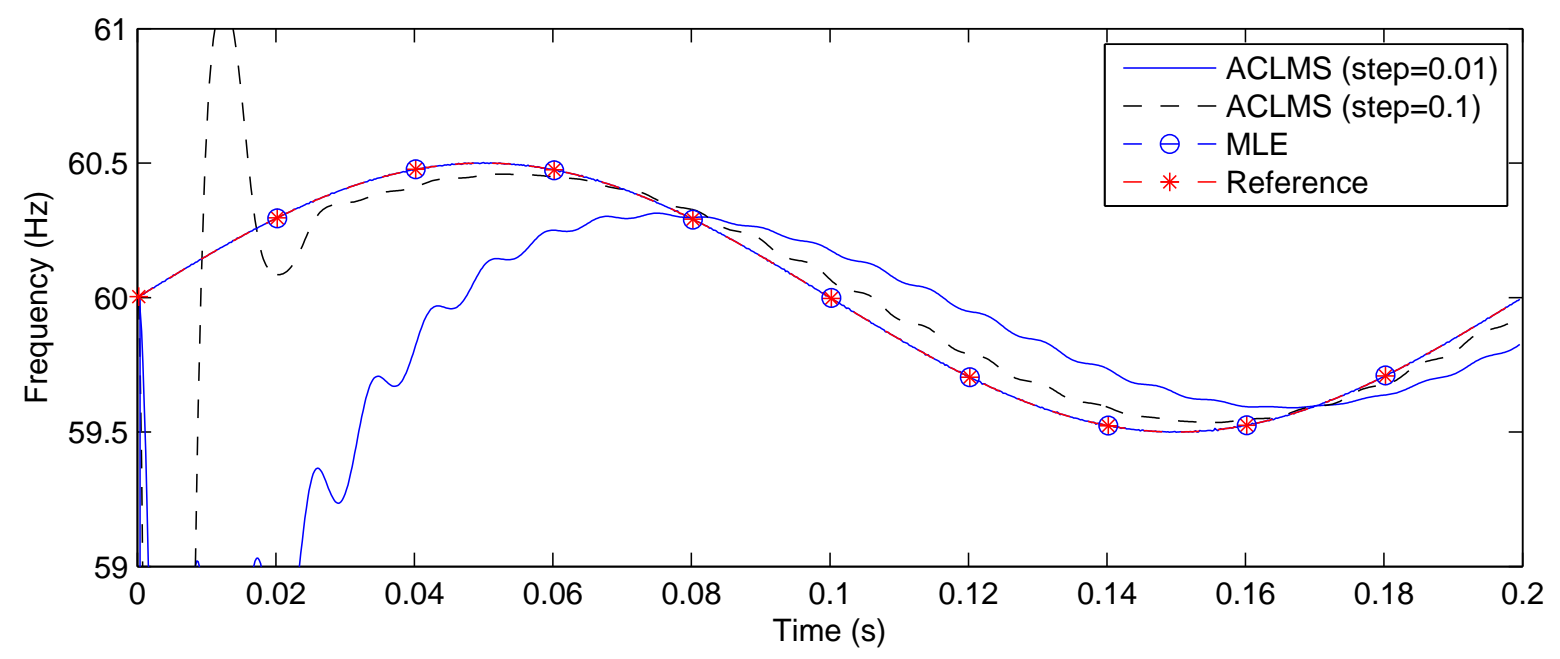

(b) Sinusoidal amplitude and phase modulation $\left(k_{x}=k_{a}=0.1\right.$ and $\left.f_{m}=5 \mathrm{~Hz}\right)$

Fig. 12: Three-phase signals according to the IEEE Standard C37.118.1 with amplitude unbalance $\left(d_{1}=\right.$ $0.75, d_{2}=1.199, \sigma^{2}=0$ and $N=1500$ samples). 